\title{
A novel multi-parametric score for the detection and grading of prosthetic mitral valve obstruction in cases with different disc motion abnormalities
}

\author{
Masoud Meskin ${ }^{\mathrm{a}, \mathrm{d}}$, Annalisa Dimasia ${ }^{\mathrm{a}}$, Emiliano Votta ${ }^{\mathrm{a}}$, Michal Jaworek ${ }^{\mathrm{a}}$, Laura Fusini ${ }^{\mathrm{b}}$, Manuela \\ Muratori $^{\mathrm{b}}$, Piero Montorsi ${ }^{\mathrm{b}, \mathrm{c}}$ Emanuele Zappa $^{\mathrm{d}}$, Ilenia Epifani ${ }^{\mathrm{e}}$, Mauro Pepi ${ }^{\mathrm{b}}$, Alberto Redaelli ${ }^{\mathrm{a}}$ \\ a Department of Electronics, Information and Bioengineering, Politecnico di Milano, Milan, Italy \\ ${ }^{b}$ Centro Cardiologico Monzino IRCCS, Milan, Italy \\ ${ }^{c}$ Department of Clinical Sciences and Community Health, Università degli Studi di Milano, Milan, \\ Italy \\ ${ }^{d}$ Department of Mechanical Engineering, Politecnico di Milano, Milan, Italy \\ e Department of Mathematics, Politecnico di Milano, Milan, Italy
}




\section{Abstract}

Prosthetic Mechanical Valves (PMV) are the elective choice in Mitral Valve (MV) replacement, because of their reliability and easiness of implantation. However, these prostheses can suffer from complications, the major one being Prosthetic Mitral Valve Thrombosis (PMVT). In these cases, Transthoracic Doppler Echocardiogram (TDE) is the standard diagnostic work-up for diagnosis of valve malfunction. The American Society of Echocardiography (ASE) indicates the possible TDE-derived indexes, which can help in identifying insurgence of MV replacement complications. Unfortunately, in some cases, it is not possible to detect PMVT based on these criteria. In these cases, we speak of Doppler Silent Thrombosis (DST) and only more accurate and invasive analyses, such as fluoroscopy, allow for a correct diagnosis.

In this work, Computational Fluid Dynamic (CFD) models were implemented to simulate valve fluid dynamics in different clinical scenarios in order to improve the reliability of PMVT diagnosis based on TDE. In detail, seven mechanical valve configurations, associated to different potential thrombotic conditions (symmetric and asymmetric stenosis), were designed and tested using 5 pathological transmitral velocity profile, extracted from real TDE images; to obtain the flow rate profiles, each TDE velocity profile was scaled to yield a Mean Flow Rate (MFR) of 4, 5 and 6 I/min, respectively. As a result, $105(7 \times 5 \times 3)$ synthetic cases, accounting for different velocity profiles, MFRs and valve configurations, were simulated. TDE-derived indexes were calculated according to the ASE guidelines were extracted. Advanced statistical methods were applied to propose a new diagnostic algorithm for detecting PMVT.

Our results showed that there isn't any significant difference between symmetric and asymmetric stenosis, probe location and flow rate waveform and confirmed that the single modality diagnostic is not able to predict thrombosis in a relevant number of cases, referable to mild and mild-severe stenosis cases. To overcome the problem, a novel multi-parametric discrete score (MPDS) based on the designed diagnostic algorithm was attained and tested; the Percentage of Stenosis (POS) was predicted with an accuracy rate of $90.5 \%$. Even more interestingly, the error rate of $9.5 \%$ is related to 4 false positive cases corresponding to mild stenosis (POS=15\%) which were erroneously classified as mild-severe stenosis. No false negatives were obtained. Our results suggest that a reliable estimation must take into account the mean flow rate as well as the transmitral velocity profile in order to provide a correct diagnosis.

Keywords: Prosthetic Mechanical Valve Thrombosis - Computational Fluid Dynamic Transthoracic Doppler Echocardiography - Linear Discriminant Analysis. 


\section{Nomenclature:}

2 MV: mitral valve

3 ASE: American society of echocardiography

4 TDE: Transthoracic doppler echocardiogram

5 DST: doppler silent thrombosis

6 PMVT: prosthetic mitral valve thrombosis

7 PMMV: prosthetic mechanical mitral valve

8 MPDS: multi-parametric discrete score

9 MPG: mean pressure gradient

10 MFR: mean flow rate

11 VTI: velocity time integration

$12 \Delta \mathrm{P}_{\max }:$ Maximum transvalvular pressure gradient

13 CFD: computational fluid dynamics

14 TAR: test accuracy rate

15 TER: test error rate

16 TER-FN: test error rate-false negatives

17 EOA: Effective Orifice Area

18 POS: Percent of Stenosis

19 LDA: Linear Discriminant Analysis

20

21 


\section{Introduction}

2 More than 100 million people worldwide suffer from valvular heart disease which is a growing problem in both developed and developing countries because of degenerative valve disease and rheumatic heart disease, respectively [1,2]. Most frequently, valvular diseases affect the Mitral Valve (MV) [3], with a 1.8\% incidence on the whole population in industrially developed countries [1].

7 If untreated, MV diseases lead to heart dysfunction and in severe cases, to heart failure or death.

8 Hence, MV diseases are typically treated through surgery.

$9 \quad$ MV repair is the procedure of choice in the US [4]. Out of 106,000 operations monitored yearly on 10 the mitral or aortic valve, the frequency of MV repair constantly increased [5-7]. Yet, when the 11 preservation of valve is not viable Mitral Valve MV replacement is needed $[8,9]$. In Europe, 12 approximately 50,000 valve replacement operations are performed annually [10]. Most frequently, Prosthetic Mechanical MV (PMMV) are used in MV replacement, because of their excellent durability [11]. However, these devices can suffer from complications, the major one being Prosthetic MV Thrombosis (PMVT), which is potentially fatal and has an incidence rate of $0.4 \%$ to $6 \%$ per patient per year [12].

17 Based on current guidelines and standards, Transthoracic Doppler echocardiogram (TDE) is the 18 initial noninvasive routine diagnostic work-up for PMVT diagnosis. These guidelines also suggest some TDE-derived indexes for assessing the severity of PMVT, complemented by the corresponding normality ranges: maximum pressure gradient across the valve $\left(\Delta \mathrm{P}_{\max }<16\right.$ $\mathrm{mmHg}$ ), Mean Pressure Gradient (MPG $<5 \mathrm{mmHg}$ ), maximum velocity $\left(\mathrm{V}_{\max }<190 \mathrm{~cm} / \mathrm{s}\right.$ ), Velocity

22 Time Integration (VTI $<20 \mathrm{~cm}$ ) and Effective Orifice Area (EOA $>2.0 \mathrm{~cm}^{2}$ ). These TDE-derived 23 indexes are always measurable, but in some cases do not allow to detect PMVT. These cases are classified as Doppler Silent Thrombosis (DST), which results in PMMV malfunction and may end in fatal stroke $[13,14]$. We hypothesized two possible causes for DST:

26 i) TDE-derived parameters are affected by uncertainty, owing in particular to the variability 27 in the positioning of the probe;

28 ii) even in absence of uncertainty, the listed TDE-derived parameters do not account for 29 some feature, e.g., trans-mitral time-dependent flow rate, that has great influence on the 30 transvalvular flow. 
1 On this basis, this work aimed to 1) investigate the reasons underlying DST and 2) build a novel

2 diagnostic algorithm to improve the reliability of PMVT diagnosis based on TDE.

3 To this purpose, a high throughput Computational Fluid Dynamics (CFD) study was developed,

4 which allowed to systematically investigate a broad range of scenarios relevant to PMVT, under

5 fully controlled conditions. In each scenario, every relevant feature was known a priori and the

6 corresponding transvalvular flow field was quantified in terms of time-dependent pressure and

7 velocity fields, with precision as well as space- and time-resolution that could not be allowed for

8 by real experiments.

10 Materials and methods

\section{CFD domain}

12 PMMV Models - The 3D geometry of a Sorin Bicarbon Fitline Bileaflet PMMV (LivaNova PLC,

13 London, United Kingdom), characterized by size $25 \mathrm{~mm}$ and maximum opening angle equal to

$1460^{\circ}$, was modeled in Ansys Design Modeler (ANSYS ${ }^{\circ}$ Academic Research Mechanical, Release

15 15). Seven different valve configurations were considered: one optimally functioning configuration

16 (Sym60_60), and six stenotic configurations obtained by tilting one or both PMMV leaflets. The

17 stenotic configurations, categorized in three stenotic levels, representing moderate, mild-sever

18 and severe stenosis with a symmetric (Sym) or asymmetric (Asym) configuration of the leaflets

19 (Table 1). The three stenotic levels were also characterized by a Percentage of Stenosis (POS)

20 equal to $15 \%, 50 \%$ and $75 \%$, respectively, where

21

$$
\text { POS }=\left(1-\frac{\text { EOA }}{\text { EOA }_{\left(\text {Sym60_60) }_{-}\right.}}\right) \times 100
$$


Table 1: Seven valve configurations were considered, including one normal and three stenotic pairs. EOAs and the corresponding POS are reported.

\begin{tabular}{cc}
\hline Valve Configuration $^{1}$ & EOA $\left(\mathrm{mm}^{2}\right)$, POS (\%) \\
\hline Sym60_60 & $239.5,0$ \\
\hline Sym50_50 & $205 / 15$ \\
Asym60_35 & \\
\hline Sym35_35 & $114.6 / 50$ \\
Asym0_57 & \\
\hline Sym20_20 & \\
\hline Asym0_35 & $60 / 75$ \\
\hline
\end{tabular}

Sym50_50, Sym35_35 and Sym20_20: stenotic symmetric configurations where the leaflets

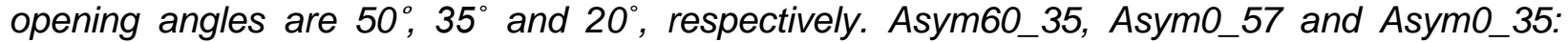
stenotic asymmetric configurations where the first number is the opening angle of the right leaflet and the second number is the opening angle of the left leaflet. The opening angles of the Asym configurations were chosen in order to have the same EOA as the Sym configurations.

Test Bench model - The geometrical model of PMMV is immersed in a fluid domain mimicking a test bench that is available at our Lab [15], which was used to run a preliminary experimental campaign on the PMMV (Supplementary material A). This choice was made so to allow for testing the reliability of the CFD model vs. experimental evidence. The 3D model of the test bench (Figure 1), was generated in Ansys Design Modeler (ANSYS ${ }^{\circledR}$ Academic Research Mechanical, Release 15). It comprises the valve housing, the atrial and ventricular chambers, an inlet conduit transversal to the atrial chamber and an outlet conduit, corresponding to the piston-pump 
connection in the experimental set-up. A flow straightener made of porous material is located at

2 the inlet of the atrial chamber, where the flow enters transversally with respect to the main flow

3 direction through the valve.

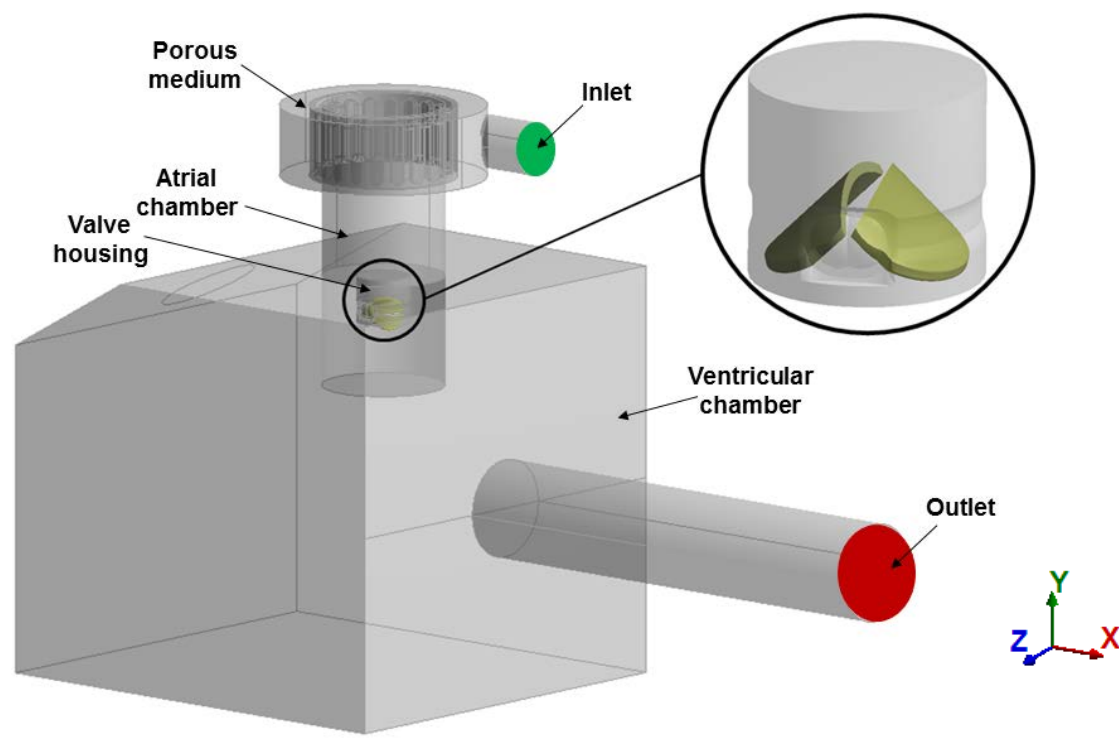

5 Figure 1: 3D model of the test bench where the different components are shown. The valve housing and leaflets are zoomed in. The leaflets here represent Sym20_20 configuration.

\section{Numerical set-up}

9 CFD transient analyses were run by means of Fluent software (ANSYS $®$ Academic Research Mechanical, Release 15). The walls of the fluid domain and the PMMV were assumed rigid and

11 a no-slip condition was applied at the walls and at the PMMV leaflets surface. The porous material

12 filling the flow straightener was modeled as an effective porous media with permeability and 13 inertial coefficients $\left(2.13 \times 10^{-10} \mathrm{~m}^{2}\right.$ and $172271 \mathrm{~m}^{-1}$, respectively) derived from experimental 14 characterizations. The working fluid was modeled as Newtonian and incompressible, with 15 isothermal rheological properties of blood at $37^{\circ} \mathrm{C}$ (with density of $1060 \mathrm{~kg} / \mathrm{m} 3$ and dynamic 16 viscosity of 0.003 Pa.s). At the outlet of the domain, a zero-constant pressure condition and at 17 the inlet, five different flow rate profiles were imposed. These five flow rate profiles were generated 18 based on transmitral time-dependent velocity profiles, obtained at Centro Cardiologico Monzino 19 IRCCS (Milan, Italy), through TDE on five PMMV recipients, characterized by different 20 hemodynamic conditions (Figure 2). These specific flow rate profiles were selected in order to 
cover a reasonably wide spectrum of diastolic hemodynamic conditions in terms of i) number of flow rate peaks (i.e., one peak only or two peaks), ii) time-delay between the two flow rate peaks, if present, and iii) slope of the waveform during the acceleration and deceleration phases.
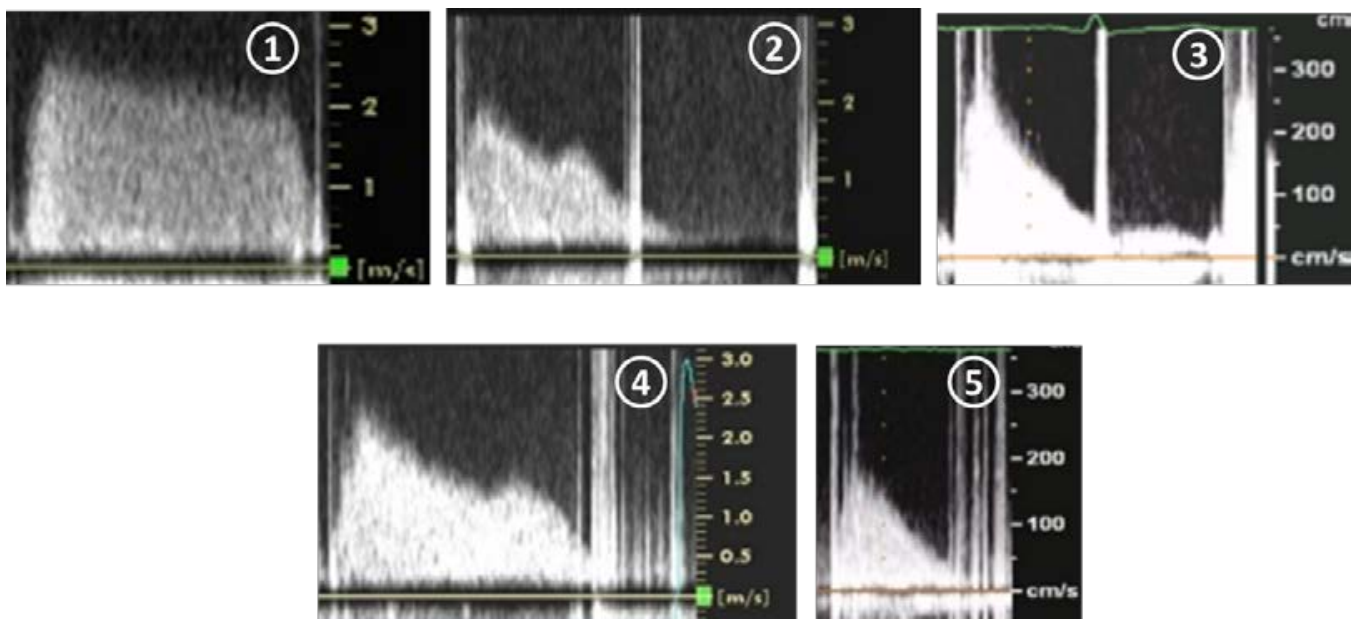

Figure 2: TDE images of velocity profiles from five cases of PMMV recipients.

In order to create the flow rate profiles, each TDE velocity profile was scaled to yield a Mean Flow Rate (MFR) of 4, 5 and $6 \mathrm{l} / \mathrm{min}$, respectively. As a result, 15 flow rate profiles (i.e., $3 \mathrm{MFR} \times 5$ transmitral velocity profile) were generated. Then, every single valve configurations were simulated with every single flow rate profiles, resulting in 105 simulations (i.e., 15 flow rate profiles $\times 7$ valve configurations).

\section{CFD data processing}

Every $1 \mathrm{~ms}$ over the simulated diastolic time, two variables were extracted:

- Transvalvular pressure gradient - two planes parallel to the valve orifice plane (normal vector is in y direction) were positioned $10 \mathrm{~mm}$ upstream $\left(\pi_{\mathrm{up}}\right)$ of the valve and $7 \mathrm{~mm}$ downstream ( $\pi_{\text {down }}$ ) of the valve (Figure 3 ). Over each plane, the area-weighted average of static pressures was computed and subsequently, $P_{\text {up }}(t)$ and $P_{\text {down }}(t)$ were obtained for the whole diastolic time. Then the time-dependent transvalvular pressure gradient was calculated as $\Delta \mathrm{P}(\mathrm{t})=\mathrm{P}_{\mathrm{up}}(\mathrm{t})-\mathrm{P}_{\text {down }}(\mathrm{t})$. Furthermore, $\mathrm{MPG}$ and $\Delta \mathrm{P}_{\max }$ were computed as:

$\mathrm{MPG}=\frac{1}{\mathrm{~N}} \int_{\mathrm{o}}^{\mathrm{t}_{\mathrm{ED}}} \Delta \mathrm{P}(\mathrm{t}) \mathrm{dt} \cong \frac{1}{\mathrm{~N}} \sum_{\mathrm{i}=1}^{\mathrm{N}} \Delta \mathrm{P}\left(\mathrm{t}_{\mathrm{i}}\right) \cdot \Delta \mathrm{t}_{\mathrm{i}}$

$\Delta \mathrm{P}_{\max }=\max \left\{\Delta \mathrm{P}\left(\mathrm{t}_{\mathrm{i}}\right)\right\}$ 
where $t_{E D}$ is the time-extent of the simulated diastolic phase and $\mathrm{N}$ is the number of timesteps at which data were extracted.

$\mathrm{VTI}=\int_{0}^{\mathrm{t}_{\mathrm{ED}}} \mathrm{V}_{\max }(\mathrm{t}) \mathrm{dt}$

21 Among the $\mathrm{VTI}, \mathrm{MPG}$ TDE and $\triangle \mathrm{P}_{\text {max-TDE }}$ values obtained for the different slices, the highest lateral 22 and central values were selected. It is worth mentioning that the central values were always 23 highest compared to the lateral ones.

24 


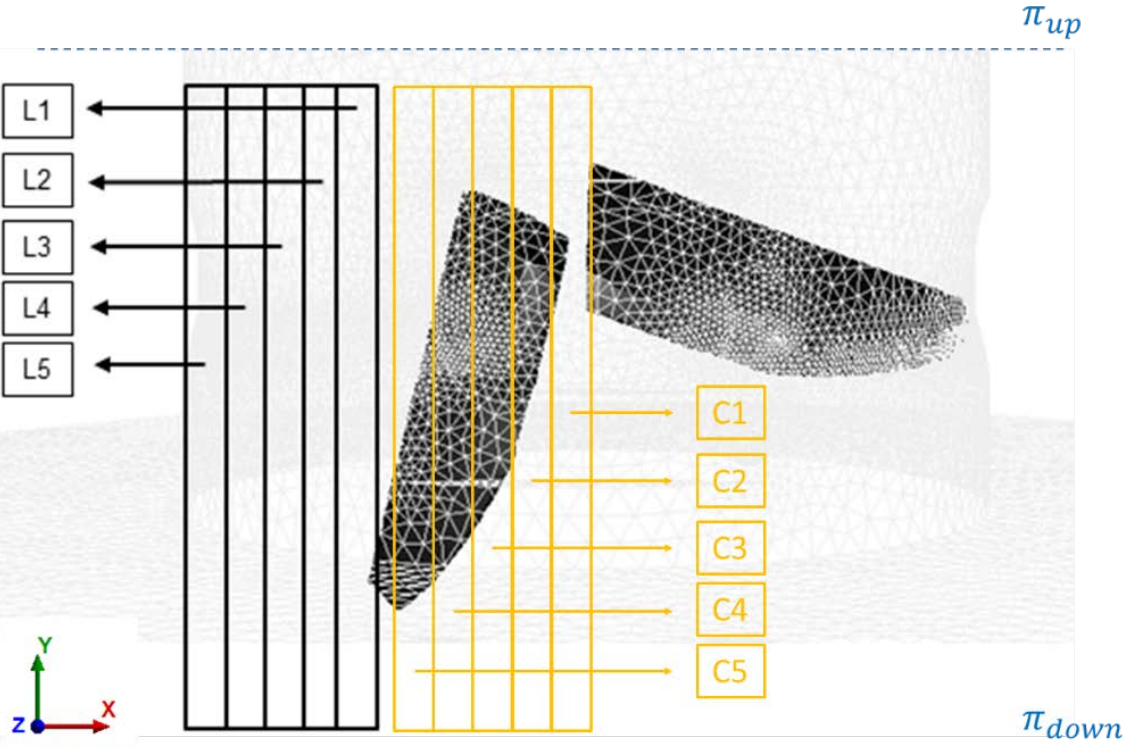

Figure 3: Orifice divisions for SAC57 configuration. Left) Lateral orifice division. Right) Central orifice division.

\section{Definition of a new multi-parametric discrete score for PMVT diagnosis}

A multi-parametric discrete score (MPDS) was defined through statistical classification of the doppler-like measures yielded by CFD simulations. The MPDS is an estimator of POS, which was built through the Linear Discriminant Analysis (LDA) $[16,17]$ of four TDE-derived indexes: MFR, $\mathrm{VTI}, \mathrm{MPG}$ and $\triangle \mathrm{P}_{\max }$. The LDA classifier is aimed at diagnosing the POS of a new patient by classifying him/her in one of four classes. The LDA model was trained using the data yielded by the simulations with the flow rate profiles of PMMV recipients 1-3 (Training Dataset, consisting of data from 63 simulations). The MPDS was then validated vs. the data yielded by the remaining simulations, i.e., those with the flow rate profiles of PMMV recipients 4 and 5 (Test Data Set, consisting of data from 42 simulations). Of note, the size of the training dataset (63 cases) may be limited for the LDA model; however, it is above the lower acceptable threshold [16, 18, 20] or close to $[16,19]$ what suggested by literatures. All statistical computations were performed with $R$ statistical software ( $R$ Foundation for Statistical Computing, Vienna, Austria) [21]. Further details are provided in Supplementary material B.

\section{Results}

The CFD results in terms of velocity contours are exemplified in Figure 4. Detailed numerical data are provided in Tables 2 to 7 . All the cases corresponding to a stenotic condition not diagnosed 
1 by any of the indexes routinely used in clinics, due to the fact that the value falls within the

2 normality range, are identified with an asterisk. In detail, Tables 2 and 3 show the maximum

3 pressure drop as calculated with the Bernoulli formula applied to the central orifice $\left(\Delta \mathrm{p}_{\max -\mathrm{TDE}}\right)$ and

4 the corresponding velocity time integration (VTI), respectively. Tables 4 and 5 allow to compare

5 the mean pressure gradient (MPG) calculated with the Bernoulli formula using the maximum

6 velocity value in the central orifice (MPG ${ }_{T D E}$ - central) and the maximum pressure gradient as

7 calculated directly with the CFD simulations (MPG). Evident strong linear correlation (with

8 coefficient of determination R-squared equal to 0.994) was found between MPG

9 MPG (Figure 5a).

10 Furthermore, in order to measure the agreement between the two methods, Bland-Altman plot of

11 the difference between MPG and MPG

12 Altman analysis (Figure $5 \mathrm{~b}$ ) highlighted a mean bias equal to $-1.9 \mathrm{mmHg}$ between the two

13 measurements. This value is consistent with the intra- and inter-operator variability previously

14 reported for peak velocity measurements through Doppler ultrasound [23]. The 95\% Limit of

15 Agreement (LOA) interval was $(-5.6 \mathrm{mmHg}$ to $1.8 \mathrm{mmHg})$; all the points in the scatterplot were 16 within the LOA, with the exception of those obtained for MPG $\geq 30 \mathrm{mmHg}$, i.e., of those 17 corresponding to clinical conditions were the diagnosis is not affected by measurement errors.

18 Tables 6 and 7 provide a comparison between the estimated values obtained by sampling from 19 the central and lateral jets; Table 6 shows the mean pressure gradient (MPG) calculated with the 20 Bernoulli formula using the maximum velocity value in the lateral orifice (MPG 21 Table 7 provides the difference between the two (Table 4 vs. Table 6). 


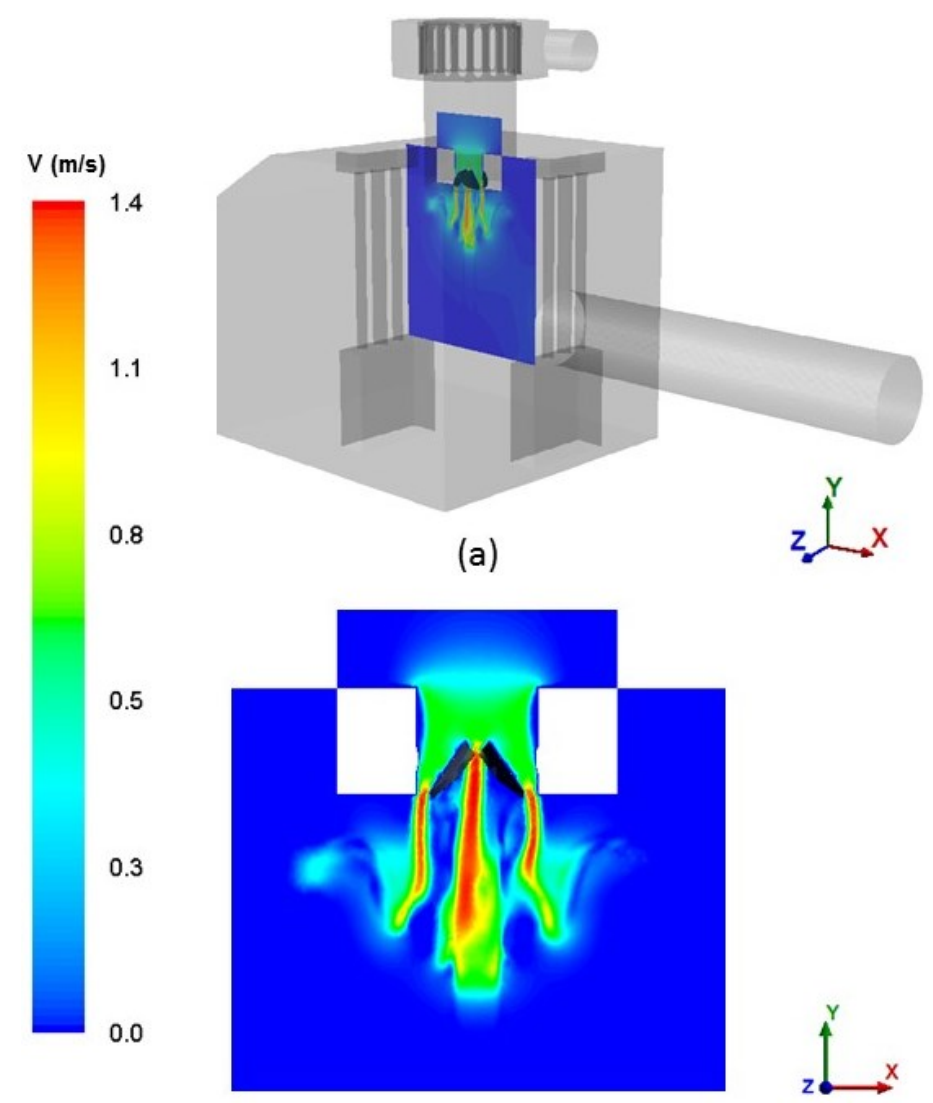

(b)

2 Figure 4: Space distribution of velocity magnitude at peak diastolic flow rate for the SS35 3 configuration with flow rate profile extracted from PMMV recipient 1 and MFR of 5 I/min. a) 4 Symmetry plane of the valve housing and leaflets, where data are exemplified. b) contour map of 5 velocity magnitude on the plane as seen in $2 D$.

6

7

8

9

10

11 


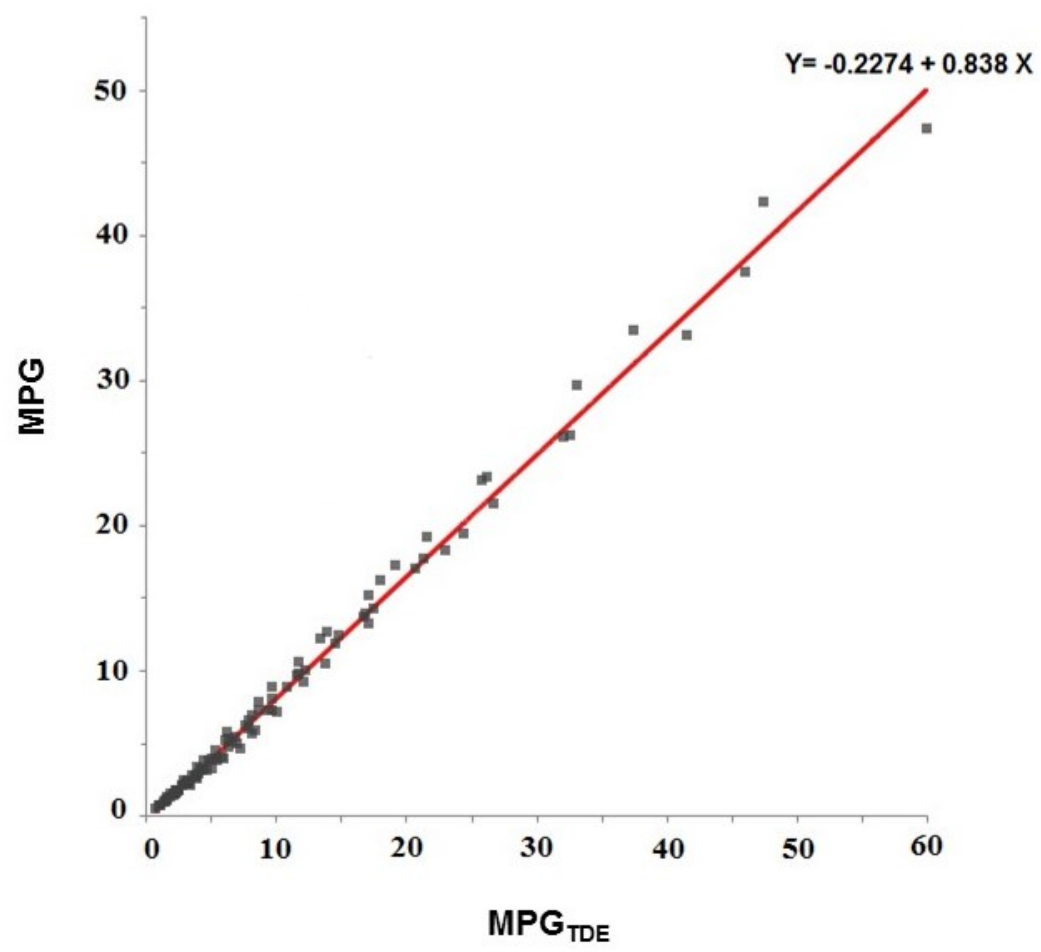

(a)

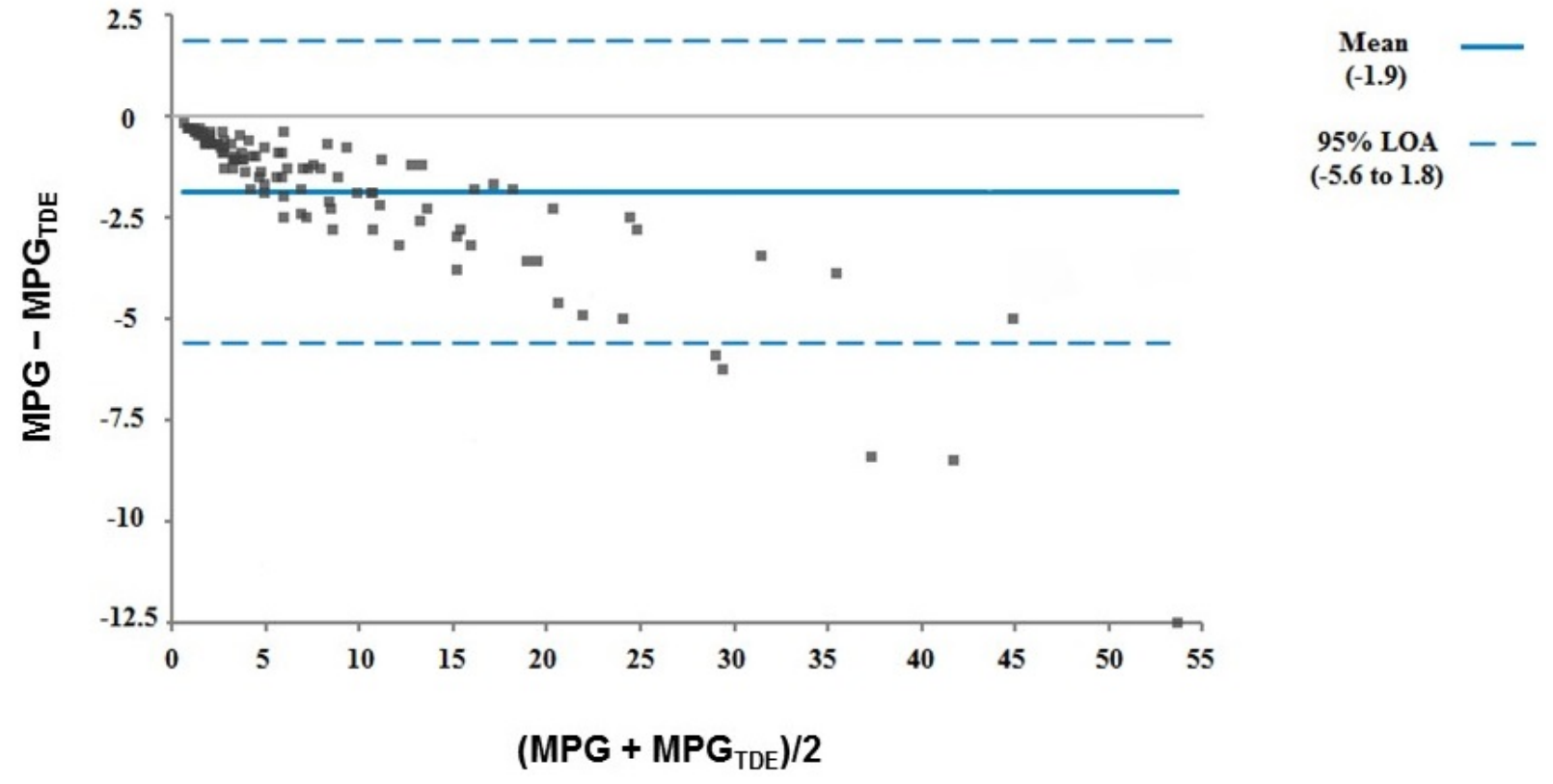

(b)

2 Figure 5: a) Regression line of MPG vs. MPG

b) Bland-Altman plot of the differences

3 between MPG and MPG ${ }_{T D E}$ against their average. 
Table 2: Maximum pressure drop calculated by the 1 Bernoulli formula for the central orifice in the 105 analyzed scenarios. Asterisks indicate

2 stenotic condition cases with $\triangle P_{\text {max-TDE }}$ values in the normality range.

Table 2: $\Delta \mathbf{P}_{\max -\mathrm{TDE}}(\mathrm{mmHg})$

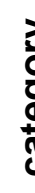

Case \& configuration

MFR (L/min)

\section{4}

5

6

\begin{tabular}{|c|c|c|c|c|c|}
\hline \multirow{21}{*}{ 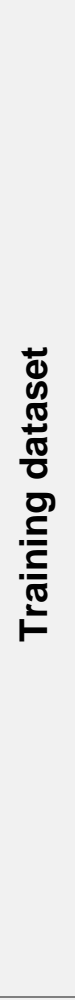 } & \multirow{7}{*}{ 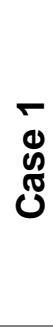 } & Sym60_60 & 2.2 & 2.7 & 3.2 \\
\hline & & Sym50_50 & $3.3^{*}$ & $4.1^{*}$ & $4.9^{*}$ \\
\hline & & Asym60_35 & $3.3^{*}$ & $4.2^{*}$ & $5.1^{*}$ \\
\hline & & Sym35_35 & $3.4^{*}$ & $4.6^{*}$ & $6.3^{*}$ \\
\hline & & Asym0_57 & $3.9 *$ & $6.1^{*}$ & $8.7^{*}$ \\
\hline & & Sym20_20 & 9.0 * & $13.9 *$ & 19.9 \\
\hline & & Asym0_35 & $10.4^{*}$ & 16.0 & 22.8 \\
\hline & \multirow{7}{*}{ 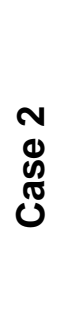 } & Sym60_60 & 2.7 & 3.5 & 4.1 \\
\hline & & Sym50_50 & $4.1^{*}$ & $5.3^{*}$ & $6.4^{*}$ \\
\hline & & Asym60_35 & $4.3^{*}$ & $5.5^{\star}$ & $7.0^{*}$ \\
\hline & & Sym35_35 & $6.0 *$ & $9.2^{*}$ & $13.2^{*}$ \\
\hline & & Asym0_57 & $8.3^{*}$ & $12.7^{*}$ & 18.0 \\
\hline & & Sym20_20 & 19.0 & 29.3 & 41.7 \\
\hline & & Asym0_35 & 21.6 & 33.1 & 46.9 \\
\hline & \multirow{7}{*}{ 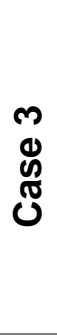 } & Sym60_60 & 4.4 & 6.8 & 10.0 \\
\hline & & Sym50_50 & $7.7^{*}$ & $11.4^{*}$ & 16.0 \\
\hline & & Asym60_35 & $8.6^{*}$ & $13.0^{*}$ & 18.6 \\
\hline & & Sym35_35 & 16.0 & 24.9 & 35.3 \\
\hline & & Asym0_57 & 21.6 & 33.1 & 46.8 \\
\hline & & Sym20_20 & 50.2 & 77.2 & 110.4 \\
\hline & & Asym0_35 & 56.3 & 86.3 & 122.6 \\
\hline \multirow{14}{*}{ 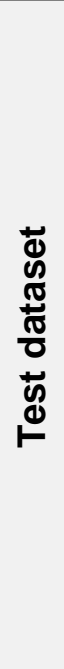 } & \multirow{7}{*}{ 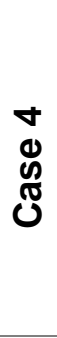 } & Sym60_60 & 3.1 & 3.9 & 4.8 \\
\hline & & Sym50_50 & $4.7^{*}$ & $6.0^{*}$ & $7.2^{*}$ \\
\hline & & Asym60_35 & $4.8^{*}$ & $6.1^{*}$ & $7.4^{*}$ \\
\hline & & Sym35_35 & $5.6^{*}$ & $8.5^{\star}$ & $12.2^{*}$ \\
\hline & & Asym0_57 & $7.7^{*}$ & $11.8^{*}$ & 16.6 \\
\hline & & Sym20_20 & 17.4 & 26.9 & 38.3 \\
\hline & & Asym0_35 & 19.9 & 30.4 & 43.1 \\
\hline & \multirow{7}{*}{ 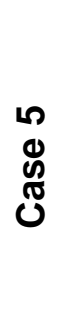 } & Sym60_60 & 4.3 & 5.8 & 7.8 \\
\hline & & Sym50_50 & $7.0^{*}$ & $9.6^{*}$ & $12.5^{\star}$ \\
\hline & & Asym60_35 & $7.7^{*}$ & $10.5^{\star}$ & $14.1^{*}$ \\
\hline & & Sym35_35 & $12.1^{*}$ & 18.8 & 26.7 \\
\hline & & Asym0_57 & 16.7 & 25.5 & 35.8 \\
\hline & & Sym20_20 & 38.3 & 59.1 & 84.2 \\
\hline & & Asym0_35 & 43.2 & 65.9 & 93.9 \\
\hline
\end{tabular}

Table 3: Velocity time integration measured in the central orifice in 105 analyzed scenarios. Asterisks indicate stenotic condition cases with VTI values in the normality range.

\section{Table 3: VTI (cm)}

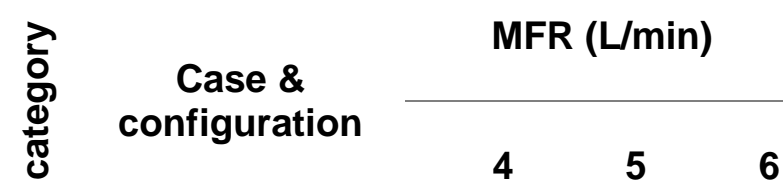

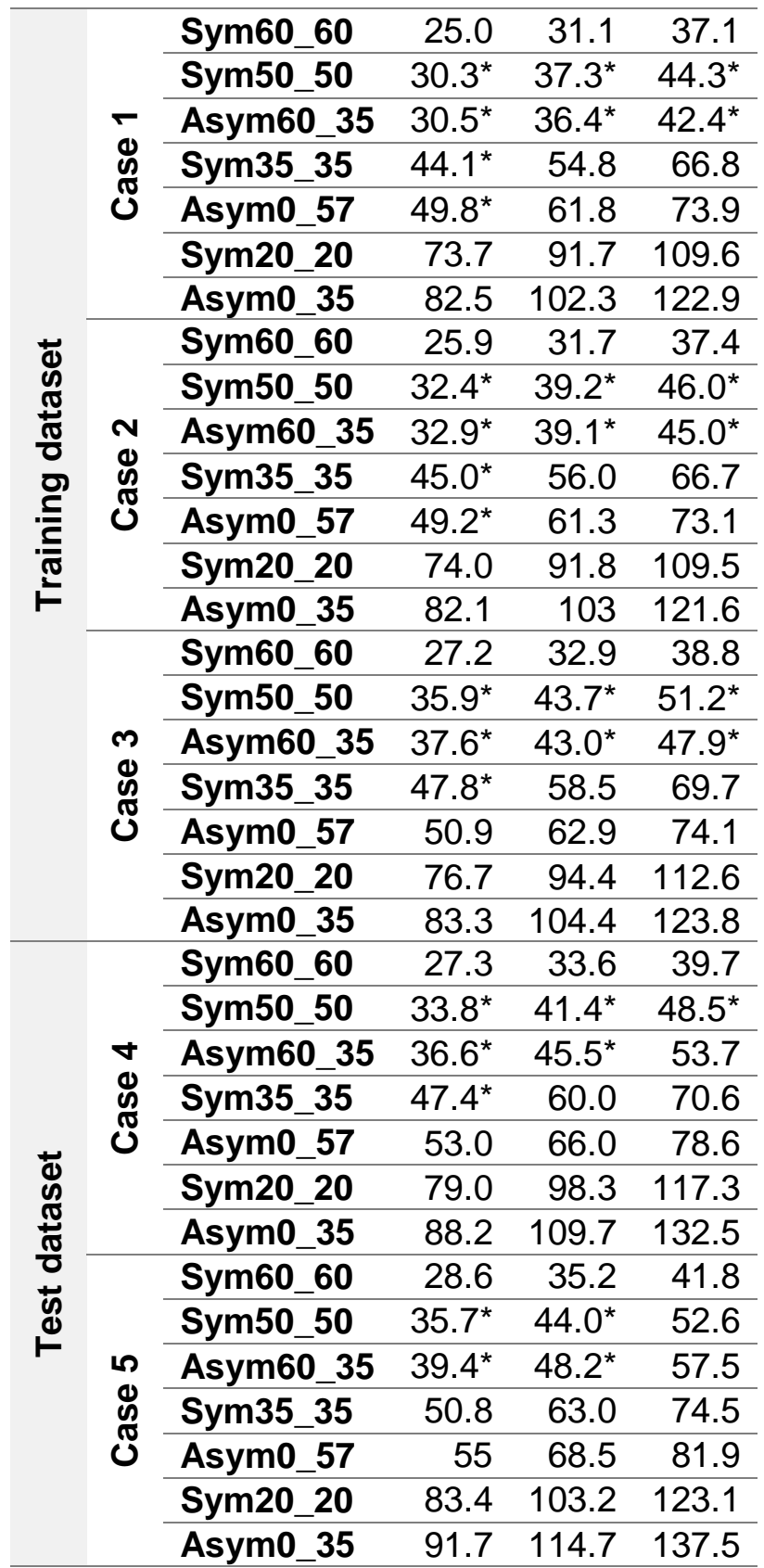


Table 4: Mean Pressure Gradient calculated by the Bernoulli formula for the central orifice in

1 the 105 analyzed scenarios. Asterisks

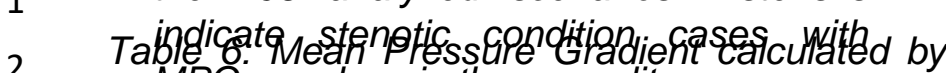

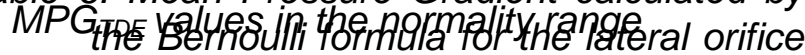

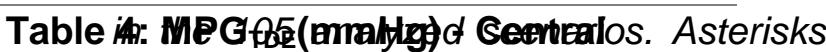
indicate stenotic condition cases with $M P G_{T D E}$ values in the normality range

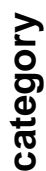

MFR (L/min)

Case \& configuration

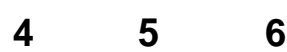

\begin{tabular}{|c|c|c|c|c|c|}
\hline \multirow{21}{*}{ 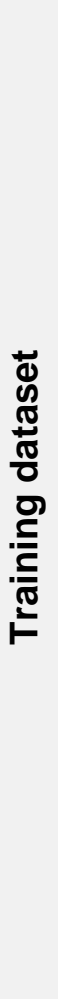 } & \multirow{7}{*}{ 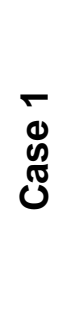 } & Sym60_60 & 0.7 & 1.1 & 1.6 \\
\hline & & Sym50_50 & $1.1^{\star}$ & $1.6^{*}$ & $2.3^{*}$ \\
\hline & & Asym60_35 & $1.3^{\star}$ & $1.9^{*}$ & $2.8^{\star}$ \\
\hline & & Sym35_35 & $2.2^{\star}$ & $3.5^{*}$ & 5.0 \\
\hline & & Asym0_57 & $2.9^{*}$ & $4.4^{*}$ & 6.3 \\
\hline & & Sym20_20 & 6.2 & 9.7 & 13.9 \\
\hline & & Asym0_35 & 7.9 & 12.2 & 17.5 \\
\hline & & Sym60_60 & 1.4 & 2.1 & 3.0 \\
\hline & & Sym50_50 & $2.2^{*}$ & $3.2^{*}$ & $4.4^{\star}$ \\
\hline & & Asym60_35 & $2.5^{\star}$ & $3.9^{*}$ & 5.4 \\
\hline & & Sym35_35 & $4.2^{\star}$ & 6.6 & 9.4 \\
\hline & & Asym0_57 & 5.3 & 8.1 & 11.6 \\
\hline & & Sym20_20 & 11.7 & 18.0 & 25.7 \\
\hline & & Asym0_35 & 14.5 & 22.9 & 32.0 \\
\hline & \multirow{7}{*}{ 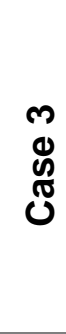 } & Sym60_60 & 2.1 & 3.1 & 4.3 \\
\hline & & Sym50_50 & $3.4^{*}$ & 5.1 & 7.2 \\
\hline & & Asym60_35 & $3.9^{*}$ & 5.9 & 8.1 \\
\hline & & Sym35_35 & 6.3 & 9.6 & 13.7 \\
\hline & & Asym0_57 & 7.6 & 11.7 & 16.8 \\
\hline & & Sym20_20 & 17.0 & 26.2 & 37.4 \\
\hline & & Asym0_35 & 20.7 & 32.5 & 46.0 \\
\hline \multirow{14}{*}{ 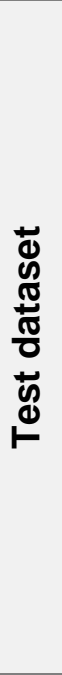 } & & Sym60_60 & 1.0 & 1.6 & 2.2 \\
\hline & & Sym50_50 & $1.5^{\star}$ & $2.3^{*}$ & $3.2^{\star}$ \\
\hline & & Asym60_35 & $1.8^{\star}$ & $2.8^{\star}$ & $4.0^{*}$ \\
\hline & & Sym35_35 & $3.1^{*}$ & $4.8^{\star}$ & 6.8 \\
\hline & & Asym0_57 & $3.9^{*}$ & 6.1 & 8.6 \\
\hline & & Sym20_20 & 8.6 & 13.4 & 19.1 \\
\hline & & Asym0_35 & 10.8 & 16.7 & 24.4 \\
\hline & \multirow{7}{*}{ 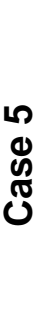 } & Sym60_60 & 2.5 & 3.8 & 5.4 \\
\hline & & Sym50_50 & $3.8^{\star}$ & 5.8 & 8.4 \\
\hline & & Asym60_35 & $4.6^{*}$ & 7.0 & 10.0 \\
\hline & & Sym35_35 & 7.8 & 12.1 & 17.1 \\
\hline & & Asym0_57 & 9.6 & 14.8 & 21.3 \\
\hline & & Sym20_20 & 21.5 & 33.1 & 47.4 \\
\hline & & Asym0_35 & 26.6 & 41.5 & 59.9 \\
\hline
\end{tabular}

Table 5: Mean Pressure Gradient calculated directly from CFD simulations in the 105 analyzed scenarios. Asterisks indicate

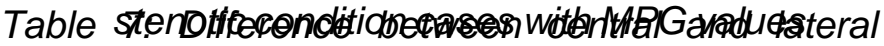

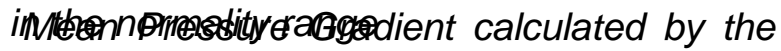

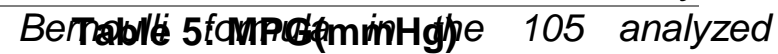
scenarios

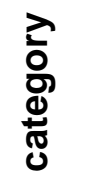

MFR (L/min) Case \&
configuration

\begin{tabular}{|c|c|c|c|c|c|}
\hline \multirow{21}{*}{ 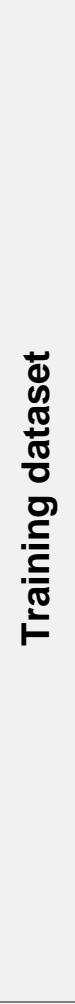 } & \multirow{7}{*}{ 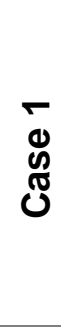 } & Sym60_60 & 0.5 & 0.8 & 1.2 \\
\hline & & Sym50_50 & $0.8^{*}$ & $1.3^{*}$ & $1.8^{*}$ \\
\hline & & Asym60_35 & $1.0^{*}$ & $1.5^{*}$ & $2.1^{*}$ \\
\hline & & Sym35_35 & $1.8^{*}$ & $2.8^{*}$ & $4.0^{*}$ \\
\hline & & Asym0_57 & $2.5^{\star}$ & $3.8^{*}$ & 5.4 \\
\hline & & Sym20_20 & 5.8 & 8.9 & 12.7 \\
\hline & & Asym0_35 & 6.6 & 10.0 & 14.3 \\
\hline & \multirow{7}{*}{$N$} & Sym60_60 & 1.0 & 1.5 & 2.2 \\
\hline & & Sym50_50 & $1.5^{\star}$ & $2.3^{*}$ & $3.3^{*}$ \\
\hline & & Asym60_35 & $1.8^{*}$ & $2.8^{*}$ & $3.9^{*}$ \\
\hline & & Sym35_35 & $3.3^{*}$ & 5.1 & 7.3 \\
\hline & & Asym0_57 & $4.5^{\star}$ & 6.9 & 9.7 \\
\hline & & Sym20_20 & 10.6 & 16.3 & 23.2 \\
\hline & & Asym0_35 & 11.9 & 18.3 & 26.1 \\
\hline & & Sym60_60 & 1.4 & 2.2 & 3.2 \\
\hline & & Sym50_50 & $2.1^{*}$ & $3.3^{*}$ & $4.7^{*}$ \\
\hline & & Asym60_35 & $2.6^{*}$ & $4.0^{*}$ & 5.7 \\
\hline & & Sym35_35 & $4.8^{*}$ & 7.3 & 10.5 \\
\hline & & Asym0_57 & 6.3 & 9.8 & 14.0 \\
\hline & & Sym20_20 & 15.2 & 23.4 & 33.5 \\
\hline & & Asym0_35 & 17.1 & 26.3 & 37.5 \\
\hline \multirow{14}{*}{ 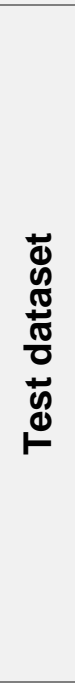 } & \multirow{14}{*}{$\begin{array}{l}5 \\
\vdots \\
0 \\
c\end{array}$} & Sym60_60 & 0.7 & 1.1 & 1.6 \\
\hline & & Sym50_50 & 1.1 & 1.7 & 2.4 \\
\hline & & Asym60_35 & $1.3^{*}$ & $2.1^{*}$ & $2.9^{*}$ \\
\hline & & Sym35_35 & $2.5^{*}$ & $3.8^{*}$ & 5.5 \\
\hline & & Asym0_57 & $3.4^{*}$ & 5.2 & 7.3 \\
\hline & & Sym20_20 & 7.9 & 12.2 & 17.3 \\
\hline & & Asym0_35 & 8.9 & 13.7 & 19.5 \\
\hline & & Sym60_60 & 1.8 & 2.8 & 4.0 \\
\hline & & Sym50_50 & $2.7^{*}$ & $4.1^{*}$ & 5.9 \\
\hline & & Asym60_35 & $3.2^{*}$ & 5.0 & 7.2 \\
\hline & & Sym35_35 & 6.0 & 9.3 & 13.3 \\
\hline & & Asym0_57 & 8.1 & 12.5 & 17.7 \\
\hline & & Sym20_20 & 19.2 & 29.7 & 42.4 \\
\hline & & Asym0_35 & 21.6 & 33.1 & 47.4 \\
\hline
\end{tabular}


Table 6: $\mathrm{MPG}_{\mathrm{TDE}}(\mathrm{mmHg})$ - Lateral

Case \&
Table 7: difference between lateral and central MPG ${ }_{T D E}(\mathrm{mmHg})$
2
응
ํㅣㅇ configuration

\begin{tabular}{|c|c|c|c|c|c|}
\hline \multirow{21}{*}{ 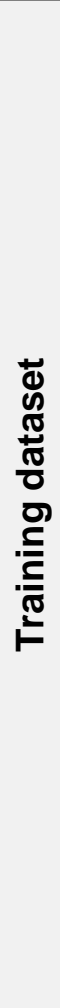 } & \multirow{7}{*}{ 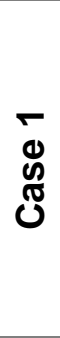 } & Sym60_60 & 0.6 & 1.0 & 1.3 \\
\hline & & Sym50_50 & $0.9 *$ & $1.4^{*}$ & $2.0 *$ \\
\hline & & Asym60_35 & $1.1^{*}$ & $1.6^{\star}$ & $2.3 *$ \\
\hline & & Sym35_35 & $2.2^{*}$ & $3.1^{*}$ & $4.4^{*}$ \\
\hline & & Asym0_57 & $2.6^{\star}$ & $3.9 *$ & 5.1 \\
\hline & & Sym20_20 & 6.0 & 9.3 & 13.3 \\
\hline & & Asym0_35 & 6.6 & 10.0 & 14.2 \\
\hline & \multirow{7}{*}{ 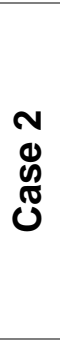 } & Sym60_60 & 1.3 & 1.9 & 2.6 \\
\hline & & Sym50_50 & $1.8^{*}$ & $2.7^{*}$ & $3.7^{*}$ \\
\hline & & Asym60_35 & $2.1^{*}$ & $3.2^{*}$ & $4.5^{*}$ \\
\hline & & Sym35_35 & $3.7^{*}$ & 5.8 & 8.2 \\
\hline & & Asym0_57 & $4.8^{*}$ & 7.1 & 10.0 \\
\hline & & Sym20_20 & 11.1 & 17.2 & 24.5 \\
\hline & & Asym0_35 & 12.0 & 18.0 & 26.3 \\
\hline & \multirow{7}{*}{ 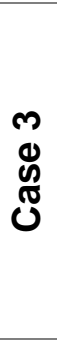 } & Sym60_60 & 2.0 & 2.9 & 3.9 \\
\hline & & Sym50_50 & $2.6 *$ & $4.0 *$ & $5.4^{*}$ \\
\hline & & Asym60_35 & $3.2^{*}$ & $4.7^{\star}$ & 6.6 \\
\hline & & Sym35_35 & 5.4 & 8.3 & 11.9 \\
\hline & & Asym0_57 & 7.1 & 10.1 & 14.9 \\
\hline & & Sym20_20 & 16.1 & 24.9 & 35.5 \\
\hline & & Asym0_35 & 17.2 & 26.1 & 37.6 \\
\hline \multirow{14}{*}{ 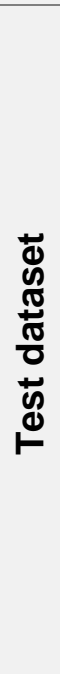 } & \multirow{7}{*}{ 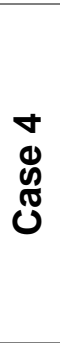 } & Sym60_60 & 0.9 & 1.4 & 1.9 \\
\hline & & Sym50_50 & $1.3 *$ & $2 *$ & $2.7^{*}$ \\
\hline & & Asym60_35 & $1.6^{*}$ & $2.4^{*}$ & $3.3 *$ \\
\hline & & Sym35_35 & $2.8 *$ & $4.3^{\star}$ & 6.2 \\
\hline & & Asym0_57 & $3.5^{*}$ & 5.3 & 7.4 \\
\hline & & Sym20_20 & 8.3 & 12.8 & 18.3 \\
\hline & & Asym0_35 & 9 & 13.8 & 19.2 \\
\hline & \multirow{7}{*}{ 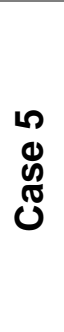 } & Sym60_60 & 2.2 & 3.3 & 4.6 \\
\hline & & Sym50_50 & $3.1^{*}$ & $4.7^{*}$ & 6.6 \\
\hline & & Asym60_35 & $3.8^{*}$ & 5.7 & 8 \\
\hline & & Sym35_35 & 6.9 & 10.6 & 15.2 \\
\hline & & Asym0_57 & 8.3 & 12.9 & 18.7 \\
\hline & & Sym20_20 & 20.4 & 31.5 & 45.1 \\
\hline & & Asym0_35 & 21.7 & 32.9 & 46.5 \\
\hline
\end{tabular}

MFR (L/min)

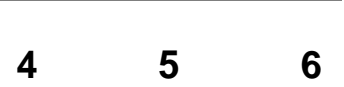

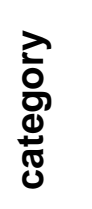

Case \& configuration
MFR (L/min)

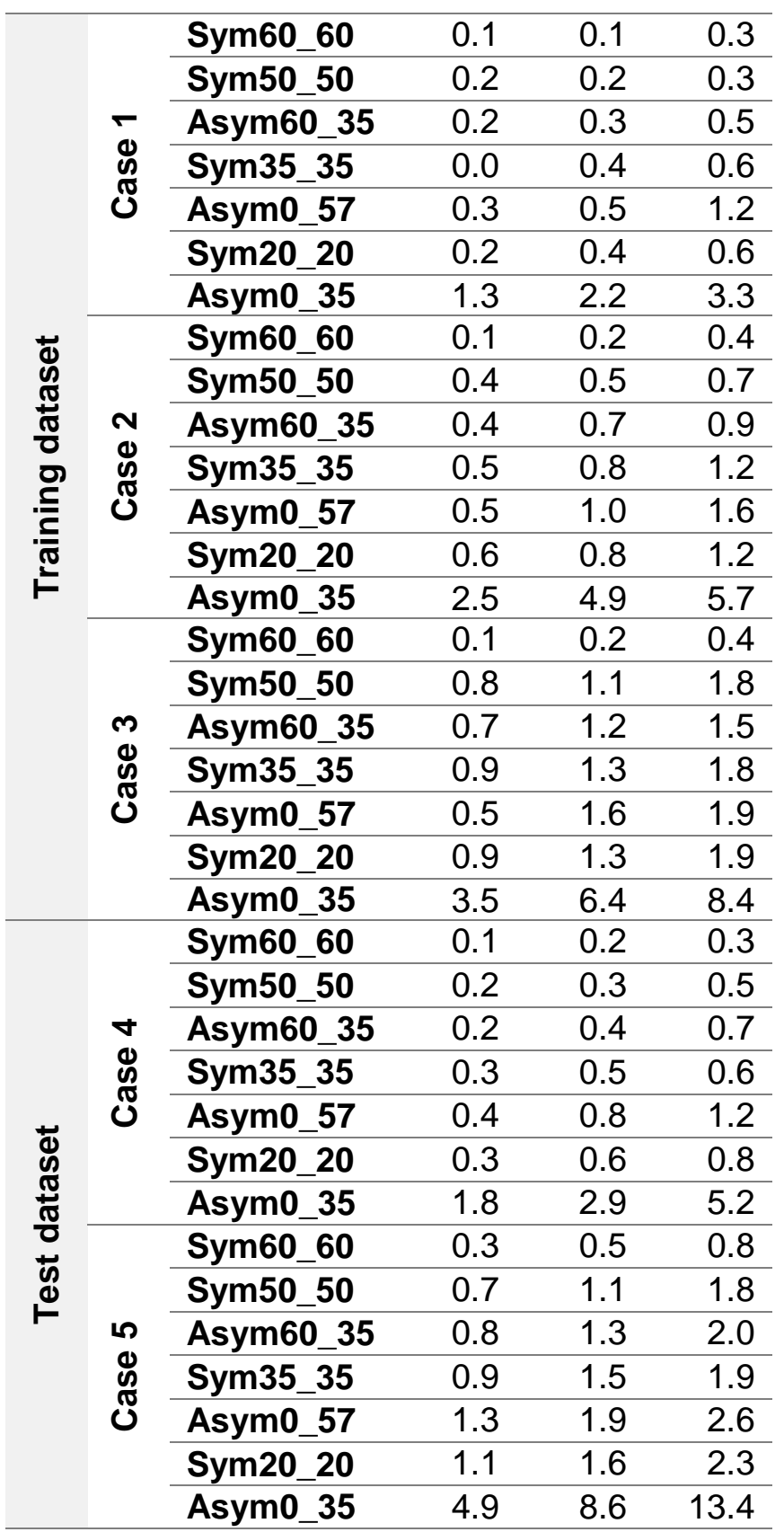


MPDS estimator - As it is mentioned before, LDA statistical method was applied to the Training

2 Dataset (cases 1-3 in Tables 2-7) and resulted in the following four linear formulas:

3

6

7

8

where $x_{1}, x_{2}, x_{3}$ and $x_{4}$ stand for MPG, MFR, $\Delta \mathrm{P}_{\max }$ and $\mathrm{VTI}$, respectively. In order to predict the POS of a new clinical case, $d_{0}, d_{15}, d_{50}$ and $d_{75}$ are computed and the case is assigned to the class which provides the highest $d_{k}$ value (see Supplementary material B for details).

The accuracy of the MPDS was assessed by building the confusion matrix obtained when applying the estimator to the Test Data Set (cases 4-5 in Tables 2-7). Details about the confusion matrix are provided in Supplementary material C. Finally, the Test Accuracy Rate (TAR, Eq.8), Test Error Rate (TER, Eq.9) and Test Error Rate resulted from False Negatives (TER-FN, Eq.10) were computed as:

$$
\mathrm{TAR}=100 \cdot \frac{\text { sum of diagonal entries }}{\text { sum of all entries }}
$$

$$
\mathrm{TER}=100 \cdot \frac{\text { sum of nondiagonal entries from lower triangle of the matrix }}{\text { sum of all entries }}
$$

$$
\text { sum of all entries }
$$

In 38 out of the 42 simulations considered in the Test Data Set, MPDS could correctly predict the POS (TAR=90.5\%). In the remaining 4 cases, a mild stenosis (POS=15\%) was predicted, even though data were from simulations replicating a normally functioning valve, leading to 4 false positives. No false negatives were obtained (TER-FN=0) and TER was equal to $9.5 \%$.

27 In order to assess the impact of each input on the predictive capability of the MPDS, four variants of the MPDS were built. For each variant, only one of the four TDE-like input indexes was dropped: 
1 the exclusion of MFR or VTI dramatically impacted POS estimation, as highlighted by the

2 confusion matrixes reported in Tables 9 and 10, respectively (further details are reported in

3 Supplementary material C). When only MFR was excluded, TAR decreased to $76.2 \%$, TER

4 increased to $23.8 \%$ and in two cases the POS was underestimated, which resulted in TER-

$5 \mathrm{FN}=4.8 \%$. The estimation was even worse when $\mathrm{VTI}$ is excluded, where TAR decreased to $47.6 \%$,

6 while TER and TER-FN increased to $52.4 \%$ and $11.9 \%$, respectively.

7 The combined effect of multiple inputs on the predictive capability of the MPDS was assessed by

8 generating 6 further variants of the MPDS, each one obtained by dropping two TDE-like input 9 indexes at a time (Supplementary material C). A spectrum of losses in predictive capability was 10 obtained. The lowest loss in predictive capability was observed when excluding both MPGTDE and $11 \Delta \mathrm{P}_{\text {max-TDE }}$ (Table 6 in Supplementary material C). When either VTI or MFR was dropped together 12 with either MPG ${ }_{T D E}$ or $\triangle P_{\max -T D E}$, the accuracy of the MPDS decreased, mostly due to an increase 13 in false negative rate (Tables 7 to 10 in Supplementary material C). The greatest loss in predictive 14 capability was recorded when dropping both VTI and MFR (Table 11 in Supplementary material $15 \mathrm{C}$ ).

Table 8: Confusion matrix on the Test Data Set comparing the predicted POS vs. the true POS 18 values.

True POS status

\begin{tabular}{c|c|cccc}
\cline { 3 - 6 } \multicolumn{2}{c|}{} & \multicolumn{5}{c}{} & 0 & 15 & 50 & 75 \\
\hline \multirow{4}{*}{$\begin{array}{c}\text { Predicted } \\
\text { POS status }\end{array}$} & 0 & 2 & 0 & 0 & 0 \\
\cline { 2 - 6 } & 15 & 4 & 12 & 0 & 0 \\
& 50 & 0 & 0 & 12 & 0 \\
\cline { 2 - 6 } & 75 & 0 & 0 & 0 & 12 \\
\hline
\end{tabular}


Table 9: Confusion matrix on the Test Data Set obtained when excluding the MFR from the MPDS estimator.

\begin{tabular}{c|c|cccc}
\multicolumn{2}{c}{} & \multicolumn{5}{c}{ True POS status } \\
\cline { 3 - 7 } \multicolumn{2}{c|}{} & 0 & 15 & 50 & 75 \\
\hline \multirow{4}{*}{$\begin{array}{c}\text { Predicted } \\
\text { POS status }\end{array}$} & 0 & 1 & 0 & 0 & 0 \\
\cline { 2 - 6 } & 15 & 5 & 10 & 2 & 0 \\
& 50 & 0 & 2 & 9 & 0 \\
\cline { 2 - 6 } & 75 & 0 & 0 & 1 & 12 \\
\hline
\end{tabular}

4

Table 10: Confusion matrix on the Test Data Set obtained after excluding the VTI from the MPDS estimator.

\section{Discussion}

10 In this paper DST was investigated by simulating different transvalvular flows and valve 11 configurations; the data obtained were used to set a novel diagnostic multi-parametric discrete 12 score based on a linear combination of TDE-derived indexes, which currently represent the gold 13 standard for diagnosing PMVT.

14 Impact of results on our initial working hypotheses - Our results prove that single modality 15 diagnostic leads to suboptimal detection of PMVT. According to data in Tables 2 to 6 , none of the 16 considered TDE-derived indexes can robustly detect the occurrence of stenosis in a standalone 17 mode. In a relevant number of simulated cases replicating a stenotic condition, indicated with the $18\left(^{*}\right)$ symbol in Tables 2 to 6 , the values of the TDE-derived indexes fell in the normality range, 
even in some of the configurations characterized by POS equal to $50 \%$ (e.g., Table 4). Instead,

2 the MPDS proved able to predict the valve POS without any false negatives and with very high accuracy.

4 This outcome did not depend significantly on the valve configuration (symmetric vs. asymmetric).

5 It has been argued in the past that an asymmetric stenosis can prevent from a correct PMVT 6 diagnosis, while being a more severe condition than symmetric stenosis [24, 25]. Instead, in this study we showed that the difference between the two conditions (Sym and Asym, results reported in Tables 2 to 6 ) is minor. This evidence strongly suggests that i) asymmetric stenosis per se cannot prevent from detecting PMVT and ii) the area of the stenosis, and not its configuration (symmetric or asymmetric) is relevant to the correct detection of PMVT.

Also, MPG ${ }_{\text {TDE }}$ values obtained when mimicking different TDE probe locations, i.e., at the central or at the lateral jets, highlighted negligible differences. These differences exceeded 3-5 $\mathrm{mmHg}$ only in those cases where the values of MPG $\mathrm{mmHg}$ ) both in the central and in the lateral jets (Table 7). Hence, these differences could not impact on diagnosis. These evidences suggest that TDE probe location cannot be the cause of DST.

Potential clinical relevance of the MPDS - The MPDS is based on LDA and linearly combines TDE-derived indexes, thus being conformal to standard clinical routine. Of note, we demonstrated that MFR information plays a key role and must be used for a correct prediction. Due to the difficulties of obtaining reliable flow rate data in mitral position, our suggestion is to estimate the MFR by measuring the stroke volume by the standard Doppler method, i.e., through the continuity equation and by measuring the LV outflow tract diameter in the parasternal long axis view and the LV outflow tract Doppler integral in the 5 Chamber view. Even more interestingly, VTI and hence the trans-mitral flow rate waveform plays a crucial role. In this work, 5 different pathological velocity profiles were simulated, with or without the A peak, and the results show that the POS estimation is affected by these data.

27 It may be argued that the approach herein proposed could be replaced by the direct analysis of the POS. In line of principle, POS could be directly estimated by obtaining the open geometry of the prosthetic leaflets through medical imaging, in light of the fact that disc opening may predict the result of thrombolysis in mitral prosthetic valve thrombosis [24]. However, even though the complete echocardiographic imaging of prosthetic heart valve includes the use of multiple views 
1 angles can be identified in $77 \%$ of patients by trans-thoracic echocardiography [25]. This

2 percentage increases to $100 \%$ when using transesophageal echocardiography, which is however

3 an invasive procedure. Cine-fluoroscopy could be a further alternative; yet, in this case radiations

4 would be a disadvantage and only 2D images would be available on a plane whose position with

5 respect to the anatomy of the patient would not be precisely known. As a result, the information

6 yielded by cine-fluoroscopy could be insufficient to assess the 3D configuration of the prosthetic

7 leaflets.

8 Summarizing, the approach herein proposed could provide more complete and reliable information on the impact of prosthetic leaflet diastolic motion on the trans-mitral hemodynamics, without the need for invasive imaging acquisitions nor for the use of radiations.

\section{Limitations}

In the present study we developed a novel MPDS to classify the severity of POS prosthetic mitral valve recipients, ultimately aiming to improving PMVT detection. The MPDS was based on a LDA model, which was identified by exploiting the data from CFD simulations of blood fluid dynamics through a mechanical heart valve within an in vitro setup. The use of CFD as a mean to systematically analyze the role of different factors in detecting PMVT and in DST allows for some advantages, which were summarized in the Introduction section, but contextually introduces some simplifying assumptions. Some of these are worth some considerations in light of the goals of the present study. In particular, standard values were set for blood density and viscosity. However, as far as density is concerned, this choice is supported by the small variability in blood density (range: $1.0239 \mathrm{~g} / \mathrm{cm}^{3}-1.0773 \mathrm{~g} / \mathrm{cm}^{3}$ ) observed in different red blood cells suspensions with of highly variable hematocrit (range: $14.2 \%$ - 85\%) [28]. As far as viscosity is concerned, its effect on the trans-mitral pressure drop was expected to be negligible, because the trans-valvular pressure drop is associated mostly to the energy loss due to the abrupt flow expansion as the blood flow advances from the valve orifice to the ventricular chamber. Such effect, though, depends on density and not on viscosity, as shown by the well-known Borda-Carnot equation. The negligible effect of changes in blood viscosity was also verified by running a series of extra simulations under the assumption that blood viscosity is equal to $4 \mathrm{cP}$ (Appendix D).

Moreover, as previously mentioned in the Materials and Methods section, the size of the training dataset, despite being acceptable, is rather limited and should be increased to have a sounder identification of the LDA model. Similarly, the size of theTest Data Set should be increased so to have a sounder verification of the predictive capability of the MPDS. 
1 Finally, the whole study was focused on the Sorin Bicarbon Fitline Bileaflet (size $25 \mathrm{~mm}$ ). This 2 specific device was chosen because of its widespread use [26, 27]. Yet, the MPDS in its current 3 version could be applied only to the detection of PMVT in recipients of this specific device. Hence, 4 at its current stage, our study should be considered a proof of concept. For the approach herein 5 proposed to be an applicable clinical tool, the study should be extended at least to a reasonably 6 wide subset of the many prosthetic valves available in the market, identifying the LDA model for 7 each considered device. 


\section{Supplementary material A: Validation of CFD simulations vs. in vitro experiments}

2 The CFD simulated scenarios, presented in the manuscript, were reproduced in an experimental 3 setup to test the reliability of the numerical results. Three Patient specific flow waveforms, 4 extracted from five PMMV recipients (Table A.1), and three valve configurations (one normal and 5 two stenotic) were considered. Then, the fluid velocities were measured by using Continuous 6 wave Doppler machine.

7 The tests were performed using a modified version of an in-vitro system previously designed and 8 exploited by our group [15] (Figure A.1). The PMMV (Bicarbon Fitline 25mm) was placed in a 9 mitral valve holder downstream of an atrial chamber with open-to-air preload reservoir and 10 upstream from a ventricular chamber. The leaflets of the PMMV were immobilized using acrylic 11 glue in three configurations: N60, SS35, SA57.

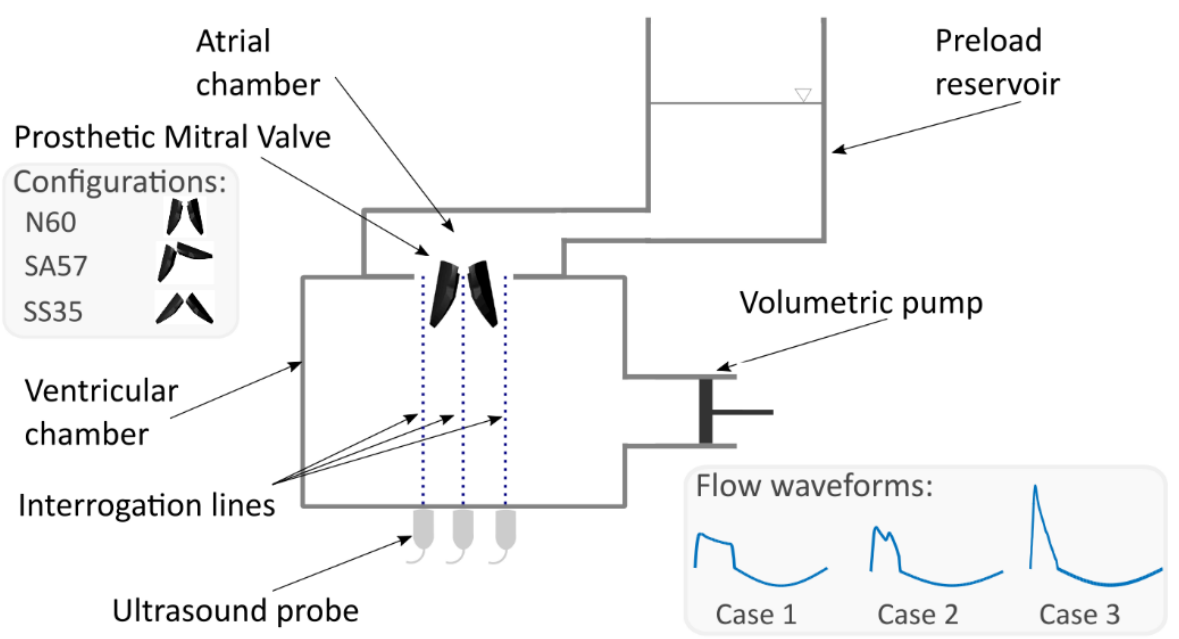

Figure A.1: Scheme of the in-vitro system.

The flow across the valve was driven by a volumetric pump which was connected to the ventricular chamber. The pump was programmed to reproduce three patient-specific diastolic flow waveforms corresponding to velocity profiles of cases 1, 2 and 3 used in CFD simulations at MFR of $5 \mathrm{~L} / \mathrm{min}$. As the study was focused on the diastolic phase only, the systolic portion of the flow waveform was simplified to a sinusoidal curve enabling transition of the fluid from the ventricular chamber back to the atrial chamber and preload reservoir (valve leaflets were immobilized). The

21 diastolic time remained as in the original waveform, whereas the systolic waveform curve was 22 scaled to obtain $2 \mathrm{~s}$ long cardiac cycle. Long systole ( 3 times longer than diastole) ensured 
1 decoupling of the dynamic effects in the two phases. The working fluid was 3.5cp glycerine

2 solution.

3 Reproduction of the imposed flow curves was verified by flow rate measurement with a transit4 time flowmeter (HT110R, Transonic System, Inc., Ithaca, NY, USA), equipped with a 1" probe

5 placed at the inflow tube between preload reservoir and the atrial chamber.

6 For each flow waveform and each valve configuration at three probe positions (27 combinations)

7 velocity profiles were acquired using Continuous wave Doppler mode of an ultrasound machine

8 (CX50 equipped with X5-1 probe, Philips, Eindhoven, The Netherlands) along three interrogation

9 lines which were axial with respect to the valve and corresponded to central and two lateral valve

10 orifice areas (Figure A.1). MPG ${ }_{T D E}, V_{\max }, V T I$ and $\Delta P_{\max }$ were computed from the experimentally-

11 derived velocity profiles.

12 Six representative measurements were confronted with CFD-derived indexes (Table A.1).

13 Representative snapshots of TDE velocity profiles and their comparison with the expected ones

14 (used in CFD simulations) are presented in Table A.2. Overall good agreement was found

15 between the experimental and CFD-derived echocardiographic indexes. The computationally

16 simulated scenarios can be assumed equivalent with the experimentally obtained ones and

17 therefore the methodology used to extract virtual TDE-derived indexes from CFD simulation can

18 be considered reliable in wide range of flow waveform morphologies and PMMV configurations.

19 Slight differences between experimental and computational outcomes can be explained by the 20 differences between the expected and measured velocity curves. E.g. if higher/lower velocity peak 21 was measured a higher/lower $\Delta \mathrm{P}_{\max }$ was obtained in respect to the corresponding computational 22 result. 
1 Table A.1: Comparison between experimentally (Exp.) and computationally (Comp.) obtained 2 echocardiographic indices.

\begin{tabular}{|c|c|c|c|c|c|c|c|c|c|c|}
\hline \multirow{2}{*}{ Case } & \multirow{2}{*}{ Configuration } & \multirow{2}{*}{$\begin{array}{l}\text { Probe } \\
\text { Position }\end{array}$} & \multirow{2}{*}{$\begin{array}{c}\begin{array}{c}\text { MPG } \\
(\mathbf{m m H g})\end{array} \\
\text { Exp. }\end{array}$} & \multicolumn{2}{|c|}{$\operatorname{Vmax}(\mathrm{m} / \mathrm{s})$} & \multicolumn{2}{|c|}{ VTI (cm) } & \multicolumn{3}{|c|}{$\Delta \mathrm{Pmax}(\mathrm{mmHg})$} \\
\hline & & & & Comp. & Exp. & Comp. & Exp. & Comp. & Exp. & Comp. \\
\hline \multirow{2}{*}{1} & \multirow{2}{*}{ SS35 } & Central & 2.3 & 3.5 & 1.3 & 1.1 & 43.4 & 54.8 & 7.2 & 5.3 \\
\hline & & Lateral & 1.4 & 3.1 & 1.2 & 1.1 & 32.3 & 52.1 & 5.5 & 4.8 \\
\hline \multirow{2}{*}{2} & \multirow{2}{*}{ N60 } & Central & 2.4 & 2.1 & 1.2 & 1.0 & 33.6 & 31.7 & 5.5 & 4.0 \\
\hline & & Lateral & 1.9 & 2.3 & 1.3 & 0.9 & 31.6 & 29.9 & 7.2 & 3.0 \\
\hline \multirow{2}{*}{3} & \multirow{2}{*}{ SA57 } & Central & 9.8 & 11.7 & 2.8 & 3.1 & 57.1 & 58.7 & 31.9 & 38.7 \\
\hline & & Lateral & 10.8 & 10.1 & 2.7 & 2.9 & 58.5 & 62.9 & 29.7 & 33.1 \\
\hline
\end{tabular}


Table A.2: Measured vs. Expected velocity profiles.
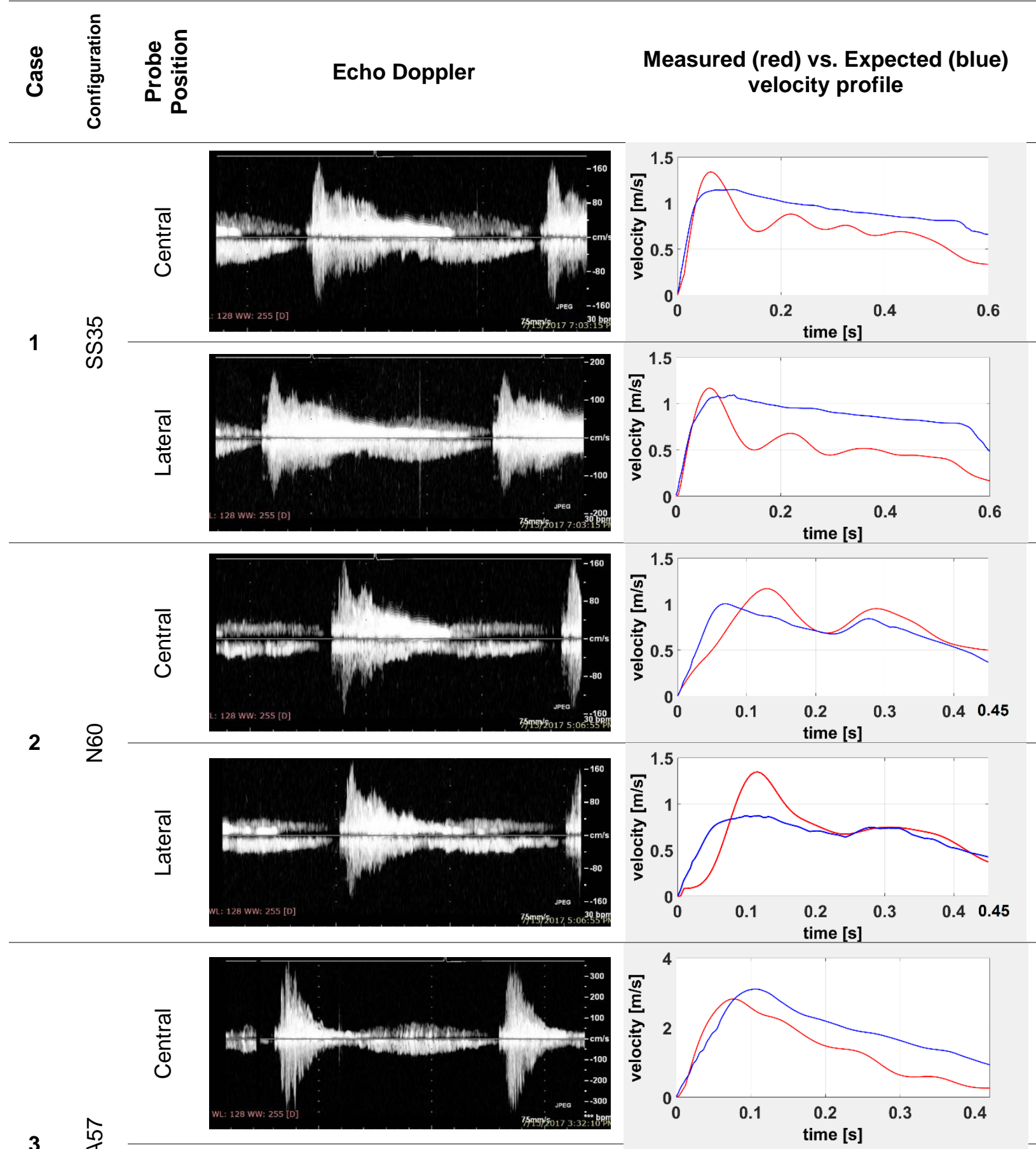

3 望
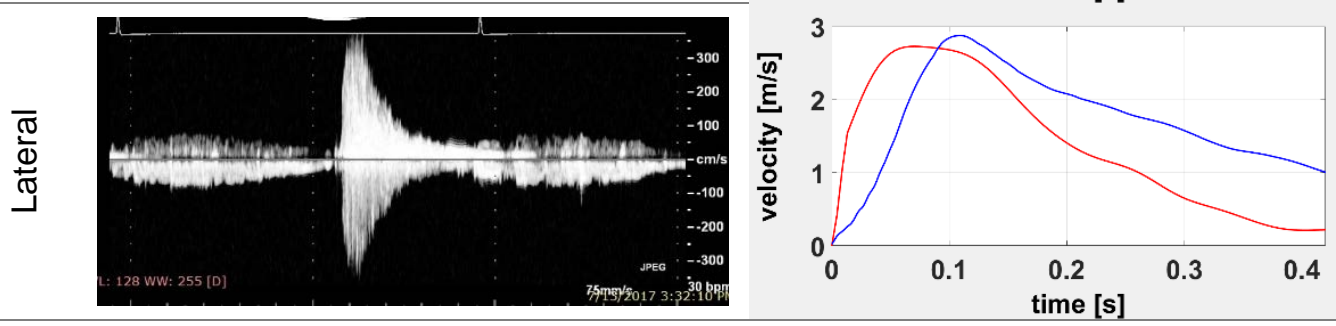


\section{Supplementary material B: the Multi-Parametric Discrete Score}

2 We applied the LDA statistical method to the Training Dataset to train the MPDS classifier to well separate the 4 stenosis classes with POS=0\%, 15\%, 50\% and 75\%. Then, MPDS was used to predict the stenosis class of Test Dataset. The MPDS classifier is a linear combination of the four discriminating variables, namely MPG, MFR, VTI and $\Delta \mathrm{P}_{\max }$.

To clarify the functionality of the MPDS classifier, we explain it in the form of pairwise "log odds ratio". For instance, for a given patient, the MPDS classifier predicts the level of stenosis equals

8 to $0 \%$ if the three pairwise "log odds ratio" (conditional on the values of MPG, MFR, $\Delta \mathrm{P}_{\max }$ and $9 \mathrm{VTI}$ ) between $0 \%$ and $15 \%$, between $0 \%$ and $50 \%$, and between $0 \%$ and $75 \%$ are all positive.

10 If one can assume that the discriminating vector (MPG, MFR, $\Delta \mathrm{P}_{\max }$ and $\mathrm{VTI}$ ) is Normal distributed 11 in each stenosis class, then all the pairwise log odds ratio of predicting level $i$ for POS (as opposed 12 to each $j \neq i$ ) are linear functions of MPG, MFR, $\Delta P_{\max }$ and VTI and the classification rule results in the following explicit formula.

For simplicity, the four discriminating variables MPG, MFR, $\Delta \mathrm{P}_{\max }$ and $\mathrm{VTI}$ are denoted by $x_{1}, x_{2}$, $x_{3}, x_{4}$, respectively. Moreover, the following statistics are observed on the Training Dataset and are involved in the MPDS classifier formula:

- $\bar{x}_{k}=\left(\bar{x}_{1 k}, \bar{x}_{2 k}, \bar{x}_{3 k}, \bar{x}_{4 k}\right)^{\prime}$ is the row vector of the group-specific sample mean of the four variables in the stenosis class POS $=k \%$ for $k=0,15,50,75$.

- $\quad S_{\mathrm{k}}$ is the sample covariance matrix of $\boldsymbol{x}$ in the $k_{t h}$ stenosis class.

- $\quad S_{\text {pooled }}$ is the pooled covariance:

where $n_{k}$ is the sample size of the $k_{\mathrm{th}}$ stenosis class in the Training Dataset (here $n_{0}=9$ and $n_{15}=\mathrm{n}_{50}$ $\left.25=\mathrm{n}_{75}=18\right)$.

26 Finally, let $p_{k}$ be the prior classification probability that a randomly chosen observation comes from 27 the class POS $=k \%$. class $k$ if the linear discriminant score: 
1

$$
d_{k}(x)=\bar{x}_{k}^{\prime} s^{-1}{ }_{\text {pooled }} x-\frac{1}{2} \bar{x}_{k}^{\prime} \bar{x}_{k}^{\prime} s^{-1}{ }_{\text {pooled }} \bar{x}_{k}+\ln p_{k}
$$

2 is the largest among all the classes. For instance, for a patient with given discriminant vector $\bar{x}_{k}$,

3 if $d_{15}(x)$ is greater with respect to $d_{0}(x), d_{50}(x)$ and $d_{75}(x)$, then the MPDS assigns that patient

4 to stenosis level $15 \%$.

5 The parameters $\bar{x}_{k}$ and $s_{\text {pooled }}$ of the MPDS classifier computed on the training set are as

6 follows:

7

$$
\bar{x}_{0}=\left(\begin{array}{c}
2.156 \\
5.000 \\
4.400 \\
32.578
\end{array}\right) \quad \bar{x}_{15}=\left(\begin{array}{c}
3.678 \\
5.000 \\
7.378 \\
39.728
\end{array}\right) \quad \bar{x}_{50}=\left(\begin{array}{c}
7.511 \\
5.000 \\
15.450 \\
59.244
\end{array}\right) \quad \bar{x}_{75}=\left(\begin{array}{c}
20.667 \\
5.000 \\
43.700 \\
97.772
\end{array}\right)
$$

8

$$
s_{\text {pooled }}=\left(\begin{array}{cccc}
42.32 & 2.68 & 122.89 & 50.08 \\
2.68 & 0.76 & 5.42 & 8.49 \\
122.89 & 5.42 & 417.09 & 106.78 \\
50.08 & 8.49 & 106.78 & 132.02
\end{array}\right)
$$

10 Finally, we chose a non-informative classification prior that assigns equal probabilities to all 11 classes of POS, i.e. $p_{k}=0.25$ for all $k=0,15 \%, 50 \%, 75 \%$. In this way we didn't include any 12 information about the prevalence of the valvular disease in the population 


\section{Supplementary material C}

2 Here we report the complete list of the confusion matrices on the Test Data Set and of the

3 corresponding values of Test Accuracy Rate (TAR), Test Error Rate (TER) and Test Error Rate

4 resulted from False Negative (TER-FN).

5 When dropping single inputs of the MPDS, the confusion matrices reported in Tables C.1-C.4

6 were obtained. The associated values of TAR, TER and TER-FN are summarized in Table C.5.

Table C.1: Confusion matrix on the Test Data Set for the MPDS without MPGTDE.

True POS status

\begin{tabular}{c|c|cccc}
\cline { 3 - 6 } \multicolumn{2}{c}{} & \multicolumn{1}{c}{0} & 15 & 50 & 75 \\
\hline \multirow{3}{*}{$\begin{array}{c}\text { Predicted } \\
\text { POS status }\end{array}$} & 0 & 2 & 0 & 0 & 0 \\
\cline { 2 - 6 } & 15 & 4 & 11 & 0 & 0 \\
\cline { 2 - 6 } & 50 & 0 & 1 & 12 & 0 \\
\cline { 2 - 6 } & 75 & 0 & 0 & 0 & 12 \\
\hline
\end{tabular}

8

Table C.2: Confusion matrix on the Test Data Set for the MPDS without $\Delta P_{\text {max-TDE. }}$

True POS status

\begin{tabular}{|c|c|c|c|c|c|}
\hline & \\
\hline & & 0 & 15 & 50 & 75 \\
\hline \multirow{4}{*}{$\begin{array}{l}\text { Predicted } \\
\text { POS status }\end{array}$} & 0 & 2 & 0 & 0 & 0 \\
\hline & 15 & 4 & 11 & 0 & 0 \\
\hline & 50 & 0 & 1 & 12 & 0 \\
\hline & 75 & 0 & 0 & 0 & 12 \\
\hline
\end{tabular}

10

Table C.3: Confusion matrix on the Test Data Set for the MPDS without MFR.

\begin{tabular}{|c|c|c|c|c|c|}
\hline & \multicolumn{4}{|c|}{ True POS status } \\
\hline & & 0 & 15 & 50 & 75 \\
\hline \multirow{4}{*}{$\begin{array}{l}\text { Predicted } \\
\text { POS status }\end{array}$} & 0 & 1 & 0 & 0 & 0 \\
\hline & 15 & 5 & 10 & 2 & 0 \\
\hline & 50 & 0 & 2 & 9 & 0 \\
\hline & 75 & 0 & 0 & 1 & 12 \\
\hline
\end{tabular}


Table C.4: Confusion matrix on the Test Data Set for the MPDS without VTI.

\begin{tabular}{c|c|cccc}
\multicolumn{2}{c}{} & \multicolumn{5}{c}{ True POS status } \\
\cline { 3 - 6 } \multicolumn{2}{c|}{} & 0 & 15 & 50 & 75 \\
\hline \multirow{4}{*}{$\begin{array}{c}\text { Predicted } \\
\text { POS status }\end{array}$} & 0 & 0 & 0 & 0 & 0 \\
\cline { 2 - 6 } & 15 & 5 & 6 & 3 & 0 \\
\cline { 2 - 6 } & 50 & 1 & 6 & 4 & 2 \\
\cline { 2 - 6 } & 75 & 0 & 0 & 5 & 10 \\
\hline
\end{tabular}

3 Table C.5: Effect of neglecting single TDE-derived indexes on the predictive capability of the MPDS.

\begin{tabular}{|c|c|c|c|c|}
\hline included & excluded & TAR (\%) & TER (\%) & TER-FN (\%) \\
\hline $\begin{array}{c}\text { MPG }_{\text {TDE }}, \Delta P_{\text {max-TDE }} \\
\text { MFR, VTI }\end{array}$ & --- & 90.5 & 9.5 & 0 \\
\hline$\Delta \mathrm{P}_{\text {max-TDE }}, \mathrm{MFR}, \mathrm{VTI}$ & MPGTDE & 88.1 & 11.9 & 0 \\
\hline MPG $_{\text {TDE, }}, \mathrm{MFR}, \mathrm{VTI}$ & $\Delta \mathrm{P}_{\text {max-TDE }}$ & 88.1 & 11.9 & 0 \\
\hline $\begin{array}{c}\text { MPG } \\
\text { TDE, } \Delta \mathrm{P}_{\max } \text {-TDE } \\
\text { VII }\end{array}$ & MFR & 76.2 & 23.8 & 4.8 \\
\hline $\begin{array}{c}\text { MPG TDE, } \Delta \mathrm{P}_{\text {max-TDE }} \\
\text { MFR }\end{array}$ & VTI & 47.6 & 52.4 & 11.9 \\
\hline
\end{tabular}

4

5 When dropping two inputs of the MPDS at a time, the confusion matrices reported in Tables C.6-

6 C.11 were obtained. The associated values of TAR, TER and TER-FN are summarized in Table C.12.

\begin{tabular}{c|c|cccc}
\multicolumn{2}{c}{} & \multicolumn{5}{c}{ True POS status } \\
\cline { 3 - 6 } \multicolumn{2}{c|}{} & 0 & 15 & 50 & 75 \\
\hline \multirow{4}{*}{$\begin{array}{c}\text { Predicted } \\
\text { POS status }\end{array}$} & 0 & 2 & 0 & 0 & 0 \\
\cline { 2 - 6 } & 15 & 4 & 11 & 0 & 0 \\
\cline { 2 - 6 } & 50 & 0 & 1 & 12 & 0 \\
\cline { 2 - 6 } & 75 & 0 & 0 & 0 & 12 \\
\hline
\end{tabular}


Table C.7: Confusion matrix on the Test Data Set for the MPDS without $\triangle \mathrm{P}_{\text {max-TDE }}$ and VTI.

True POS status

\begin{tabular}{c|c|cccc}
\cline { 3 - 6 } \multicolumn{2}{c}{} & \multicolumn{1}{c}{0} & 15 & 50 & 75 \\
\hline \multirow{3}{*}{$\begin{array}{c}\text { Predicted } \\
\text { POS status }\end{array}$} & 0 & 0 & 0 & 0 & 0 \\
\cline { 2 - 6 } & 15 & 6 & 8 & 2 & 0 \\
\cline { 2 - 6 } & 50 & 0 & 4 & 8 & 0 \\
\cline { 2 - 6 } & 75 & 0 & 0 & 2 & 12 \\
\hline
\end{tabular}

Table C.8: Confusion matrix on the Test Data Set for the MPDS without MPGTDE and MFR.

\begin{tabular}{c|c|cccc}
\multicolumn{2}{c}{} & \multicolumn{5}{c}{ True POS status } \\
\cline { 3 - 6 } \multicolumn{2}{c|}{} & 0 & 15 & 50 & 75 \\
\hline \multirow{4}{*}{$\begin{array}{c}\text { Predicted } \\
\text { POS status }\end{array}$} & 0 & 0 & 0 & 0 & 0 \\
& 15 & 6 & 9 & 1 & 0 \\
& 50 & 0 & 3 & 9 & 0 \\
\cline { 2 - 6 } & 75 & 0 & 0 & 2 & 12 \\
\hline
\end{tabular}

4

Table C.9: Confusion matrix on the Test Data Set for the MPDS without VTI and $\Delta P_{\text {max-TDE. }}$

\begin{tabular}{c|c|cccc}
\multicolumn{2}{c}{} & \multicolumn{5}{c}{ True POS status } \\
\cline { 3 - 6 } \multicolumn{2}{c|}{} & 0 & 15 & 50 & 75 \\
\hline \multirow{4}{*}{$\begin{array}{c}\text { Predicted } \\
\text { POS status }\end{array}$} & 0 & 0 & 0 & 0 & 0 \\
\cline { 2 - 6 } & 15 & 5 & 7 & 3 & 0 \\
\cline { 2 - 6 } & 50 & 1 & 5 & 7 & 0 \\
\hline & 75 & 0 & 0 & 2 & 12 \\
\hline
\end{tabular}

Table C.10: Confusion matrix on the Test Data Set for the MPDS without VTI and MPGTDE.

True POS status

\begin{tabular}{|c|c|c|c|c|c|}
\hline & & & & \\
\hline & & 0 & 15 & 50 & 75 \\
\hline \multirow{3}{*}{$\begin{array}{l}\text { Predicted } \\
\text { POS status }\end{array}$} & 0 & 0 & 0 & 0 & 0 \\
\hline & 15 & 5 & 8 & 3 & 0 \\
\hline & 50 & 1 & 4 & 9 & 3 \\
\hline
\end{tabular}


\begin{tabular}{l|llll}
75 & 0 & 0 & 0 & 9
\end{tabular}

Table C.11: Confusion matrix on the Test Data Set for the MPDS without MFR and VTI.

True POS status

\begin{tabular}{c|c|cccc}
\cline { 3 - 6 } \multicolumn{2}{c|}{} & \multicolumn{1}{c}{0} & 15 & 50 & 75 \\
\hline \multirow{4}{*}{$\begin{array}{c}\text { Predicted } \\
\text { POS status }\end{array}$} & 0 & 0 & 0 & 0 & 0 \\
\cline { 2 - 6 } & 15 & 5 & 8 & 3 & 0 \\
\cline { 2 - 6 } & 50 & 1 & 4 & 5 & 3 \\
\cline { 2 - 6 } & 75 & 0 & 0 & 4 & 9 \\
\hline
\end{tabular}

2

3 Table C.12: Effect of neglecting two TDE-derived indexes at a time on the predictive capability of the MPDS.

\begin{tabular}{|c|c|c|c|c|}
\hline included & excluded & TAR (\%) & TER (\%) & TER-FN (\%) \\
\hline MFR, VTI & $\begin{array}{l}\text { MPG }_{\text {TDE }} \\
\Delta \mathrm{P}_{\text {max-TDE }}\end{array}$ & 88.1 & 11.9 & 0 \\
\hline$\Delta \mathrm{P}_{\text {max-TDE }}, \mathrm{VTI}$ & $\begin{array}{l}\text { MPGTDE }_{\text {MFR }} \\
\text { MFR }\end{array}$ & 71.4 & 28.6 & 2.4 \\
\hline MPGTDE, VTI & $\begin{array}{c}\Delta P_{\text {max-TDE }} \\
\text { MFR }\end{array}$ & 66.7 & 33.3 & 4.8 \\
\hline $\mathrm{MPG}_{\mathrm{TDE}}, \mathrm{MFR}$ & $\Delta \mathrm{P}_{\text {max-TDE }}, \mathrm{VTI}$ & 57.4 & 42.6 & 7.1 \\
\hline$\Delta \mathrm{P}_{\text {max-TDE }}, \mathrm{MFR}$ & MPG $_{T D E}, V T I$ & 61.9 & 38.1 & 14.3 \\
\hline $\mathrm{MPG}_{\mathrm{TDE}}, \Delta \mathrm{P}_{\text {max-TDE }}$ & MFR, VTI & 52.4 & 47.6 & 14.3 \\
\hline
\end{tabular}

5

6 


\section{Supplementary material D}

2 All of the CFD simulations were setting standard values for blood viscosity $(\mu=3 \mathrm{cp})$ and density

$3\left(\rho=1060 \mathrm{~kg} / \mathrm{m}^{3}\right)$, under the hypothesis that changes in these two parameters could be small and

4 could have negligible effects on computational results. Here we report the results from an extra

5 series of CFD simulations, which were run to verify the effects of changing blood viscosity. In

6 particular, upon increasing blood viscosity to $4 \mathrm{cp}$ simulations were run again for each the four

7 prosthetic valve configurations, imposing an inlet flow rate with mean value of $5 \mathrm{l} / \mathrm{min}$ and

8 waveform consistent with recipient 3. Tables D.1-D.4 show that the change in blood viscosity had

9 no or minor impact on the results, suggesting that our initial hypothesis on blood rheological

10 properties should not affect our results.

11

Table D.1: Maximum pressure drop calculated by the Bernoulli formula ( $\triangle \mathrm{Pmax}$-TDE) for the central orifice for case 3 with MFR of 5 lit/min.

$\Delta \mathbf{P}_{\text {max-TDE }}(\mathbf{m m H g})$

\begin{tabular}{|c|c|c|c|}
\hline \multirow{3}{*}{\multicolumn{2}{|c|}{$\begin{array}{c}\text { Case \& } \\
\text { configuration }\end{array}$}} & \multirow{2}{*}{\multicolumn{2}{|c|}{$\begin{array}{c}\text { MFR = 5lit/min } \\
\text { Blood viscosity (cp) }\end{array}$}} \\
\hline & & & \\
\hline & & \multirow{2}{*}{$\begin{array}{c}3 \\
6.8\end{array}$} & \multirow{2}{*}{$\begin{array}{c}4 \\
7.0\end{array}$} \\
\hline \multirow{4}{*}{ 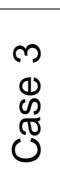 } & Sym60_60 & & \\
\hline & Asym60_35 & 13.0 & 13.2 \\
\hline & Asym0_57 & 33.1 & 38.8 \\
\hline & Asym0_35 & 86.3 & 87.4 \\
\hline
\end{tabular}

12

Table D.2: Velocity time integral (VTI) measured in the central orifice for case 3 with MFR of 5 lit/min.

Table 2: VTI (cm)

\begin{tabular}{|c|c|c|c|}
\hline \multirow{3}{*}{\multicolumn{2}{|c|}{$\begin{array}{c}\text { Case \& } \\
\text { configuration }\end{array}$}} & \multirow{2}{*}{\multicolumn{2}{|c|}{$\begin{array}{c}\text { MFR = 5lit/min } \\
\text { Blood viscosity (cp) }\end{array}$}} \\
\hline & & & \\
\hline & & 3 & 4 \\
\hline \multirow{4}{*}{ 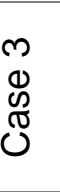 } & Sym60_60 & 32.9 & 33.0 \\
\hline & Asym60_35 & 43.0 & 43.6 \\
\hline & Asym0_57 & 62.9 & 63.1 \\
\hline & Asym0_35 & 104.4 & 105.5 \\
\hline
\end{tabular}


Table D.3: Mean Pressure Gradient (MPGTDE) calculated by the Bernoulli formula for the central orifice for case 3 with MFR of 5 lit/min.

Table 3: $\mathrm{MPG}_{\mathrm{TDE}}(\mathrm{mmHg})$ - Central

\begin{tabular}{|c|c|c|c|}
\hline \multirow{3}{*}{\multicolumn{2}{|c|}{$\begin{array}{c}\text { Case \& } \\
\text { configuration }\end{array}$}} & \multirow{2}{*}{\multicolumn{2}{|c|}{$\begin{array}{c}\text { MFR = 5lit/min } \\
\text { Blood viscosity (cp) }\end{array}$}} \\
\hline & & & \\
\hline & & \multirow{2}{*}{$\begin{array}{c}3 \\
3.1\end{array}$} & \multirow{2}{*}{$\begin{array}{c}4 \\
3.1\end{array}$} \\
\hline \multirow{4}{*}{ 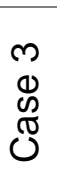 } & Sym60_60 & & \\
\hline & Asym60_35 & 5.9 & 5.9 \\
\hline & Asym0_57 & 11.7 & 11.8 \\
\hline & Asym0_35 & 32.5 & 33.2 \\
\hline
\end{tabular}

2

Table D.4: Mean Pressure Gradient (MPG ${ }_{T D E}$ ) calculated with the Bernoulli formula at the lateral orifice for case 3 with MFR of 5 lit/min.

Table 4: MPGTDE(mmHg) - Lateral

\begin{tabular}{|c|c|c|c|}
\hline & \multicolumn{2}{|c|}{ MFR = 5lit $/ \mathrm{min}$} \\
\hline \multirow{2}{*}{\multicolumn{2}{|c|}{$\begin{array}{c}\text { Case \& } \\
\text { configuration }\end{array}$}} & \multicolumn{2}{|c|}{ Blood viscosity (cp) } \\
\hline & & 3 & 4 \\
\hline \multirow{4}{*}{ 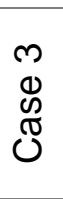 } & Sym60_60 & 2.9 & 2.9 \\
\hline & Asym60_35 & 4.7 & 4.8 \\
\hline & Asym0_57 & 10.1 & 10.5 \\
\hline & Asym0_35 & 26.1 & 26.2 \\
\hline
\end{tabular}

3 


\section{$1 \quad$ References}

2 [1] Nkomo VT, Gardin JM, Skelton TN, Gottdiener JS, Scott CG, Enriquez-Sarano M. Burden of

3 valvular heart diseases: a population-based study. Lancet, 2006; 368:1005-1011

4 [2] Carapetis JR, Steer AC, Mulholland EK, Weber M. The global burden of group A streptococcal

5 diseases. Lancet Infect Dis, 2005; 5:685-694

6 [3] Zoltan G. Turi, Mitral Valve Disease. AHA J, 2004;109:e38-e41

7 [4] Gillinov AM, Cosgrove DM, Blackstone EH, Diaz R, Arnold JH, Lytle BW, Smedira NG, Sabik

8 JF, McCarthy PM, Loop FD. Durability of mitral valve repair for degenerative disease. J Thorac

9 Cardiovasc Surg. 1998;116734-43.

10 [5] Valve Repair or Replacement, Texas Heart Institute, 2018

11 http://www.texasheart.org/HIC/Topics/Proced/vsurg.cfm

12 [6] Savage EB, Ferguson TB Jr, DiSesa VJ. Use of mitral valve repair: analysis of contemporary 13 United States experience reported to the Society of Thoracic Surgeons National Cardiac 14 Database. Ann Thorac Surg. 2003;75:820-5.

15 [7] Gammie JS, Sheng S, Griffith BP, Peterson ED, Rankin JS, O'Brien SM, Brown JM. Trends in 16 mitral valve surgery in the United States: results from the Society of Thoracic Surgeons Adult 17 Cardiac Surgery Database. Ann Thorac Surg. 2009;87:1431-7

[8] Nishimura RA, Otto CM, Bonow RO, Carabello BA, Erwin JP 3rd, Guyton RA, O'Gara PT, Ruiz CE, Skubas NJ, Sorajja P, Sundt TM 3rd, Thomas JD; American College of Cardiology/American Heart Association Task Force on Practice Guidelines. 2014 AHA/ACC guideline for the management of patients with valvular heart disease: executive summary: a report of the American

22 College of Cardiology/American Heart Association Task Force on Practice Guidelines. J Am Coll 23 Cardiol. 2014; 63:2438-88.

24 [9] Nishimura RA, Otto CM, Bonow RO, Carabello BA, Erwin JP 3rd, Fleisher LA, Jneid H, Mack 25 MJ, McLeod CJ, O'Gara PT, Rigolin VH, Sundt TM 3rd, ThoMPDSon A. 2017 AHA/ACC Focused 26 Update of the 2014 AHA/ACC Guideline for the Management of Patients With Valvular Heart 27 Disease: A Report of the American College of Cardiology/American Heart Association Task Force on Clinical Practice Guidelines. J Am Coll Cardiol. 2017; 70:252-289. 
1 [10] Butchart EG, Gohlke-Bärwolf C, Antunes MJ, Tornos P, De Caterina R, Cormier B, 2 Prendergast B, lung B, Bjornstad H, Leport C, Hall RJC, Vahanian A. Recommendations for the 3 management of patients after heart valve surgery, Europ Heart J, 2005; 26:2463-2471.

4 [11] Rahimtoola SH. Choice of prosthetic heart valve in adults an update. J Am Coll Cardiol. 2010; 5 55:2413-26.

[12] Tanis W, Habets J, van den Brink RB, Symersky P, Budde RP, Chamuleau SA. Differentiation of thrombus from pannus as the cause of acquired mechanical prosthetic heart valve obstruction by non-invasive imaging: a review of the literature. Eur Heart J Cardiovasc Imaging. 2014;15:11929.

[13] Lancellotti P, Pibarot P, Chambers J, Edvardsen T, Delgado V, Dulgheru R, Pepi M, Cosyns B, Dweck MR, Garbi M, Magne J, Nieman K, Rosenhek R, Bernard A, Lowenstein J, Vieira ML, Rabischoffsky A, Vyhmeister RH, Zhou X, Zhang Y, Zamorano JL, Habib G. Recommendations for the imaging assessment of prosthetic heart valves: a report from the European Association of Cardiovascular Imaging endorsed by the Chinese Society of Echocardiography, the InterAmerican Society of Echocardiography, and the Brazilian Department of Cardiovascular Imaging. Eur Heart J Cardiovasc Imaging. 2016;17:589-90.

[14] Zoghbi WA, Chambers JB, Dumesnil JG, Foster E, Gottdiener JS, Grayburn PA, Khandevine RA, Marx GR, Miller FA Jr, Nakatani S, Quiñones MA, Rakowski H, Rodriguez LL, Swaminathan M, Waggoner AD, Weissman NJ, Zabalgoitia M; American Society of Echocardiography's Guidelines and Standards Committee; Task Force on Prosthetic Valves; American College of Cardiology Cardiovascular Imaging Committee; Cardiac Imaging Committee of the American Heart Association; European Association of Echocardiography; European Society of Cardiology; Japanese Society of Echocardiography; Canadian Society of Echocardiography; American College of Cardiology Foundation; American Heart Association; European Association of Echocardiography; European Society of Cardiology; Japanese Society of Echocardiography; Canadian Society of Echocardiography. Recommendations for evaluation of prosthetic valves 27 with echocardiography and Doppler ultrasound: a report From the American Society of 28 Echocardiography's Guidelines and Standards Committee and the Task Force on Prosthetic 29 Valves, developed in conjunction with the American College of Cardiology Cardiovascular 30 Imaging Committee, Cardiac Imaging Committee of the American Heart Association, the 31 European Association of Echocardiography, a registered branch of the European Society of 32 Cardiology, the Japanese Society of Echocardiography and the Canadian Society of 
1 Echocardiography, endorsed by the American College of Cardiology Foundation, American Heart

2 Association, European Association of Echocardiography, a registered branch of the European

3 Society of Cardiology, the Japanese Society of Echocardiography, and Canadian Society of

4 Echocardiography. J Am Soc Echocardiogr. 2009; 22:975-1014; quiz 1082-4.

5 [15] Vismara R, Pavesi A, Votta E, Taramasso M, Maisano F, Fiore GB. A pulsatile simulator for 6 the in vitro analysis of the mitral valve with tri-axial papillary muscle displacement. Int J Artif 7 Organs. 2011; 34(4):383-391.

8 [16] Izenman AJ, Modern Multivariate Statistical Techniques. New York. Springer, 2013.

9 [17] James G, Witten D, Hastie T, Tibshirani R. An Introduction to Statistical Learning with 10 Applications in R. Springer Texts in Statistics.

11 [18] Lachenbruch PJ, Goldstein M. Discriminant Analysis. Biometrics. 1979; 35(1):69-85.

12 [19] Hair F, Black W, Babin B, Anderson R, Tatham R. Multivariate Data Analysis. 6th ed. Upper 13 Saddle River. Pearson Prentice Hall, 2006.

14 [20] Zavorka S, Perrett JJ. Minimum Sample Size Considerations for Two-Group Linear and 15 Quadratic Discriminant Analysis with Rare Populations. Communications in Statistics-Simulation 16 and Computation. 2013; 43:1726-1739.

17 [21] R Development Core Team. R: A language and environment for statistical computing. R Foundation for Statistical Computing, 2017; Vienna, Austria. ISBN 3-900051-07-0, URL http://www.R-project.org.

20

21

[22] Bland JM, Altman DG. Statistical methods for assessing agreement between two methods of clinical measurement. Lancet 1986; i:307-310.

[23] van Dijk APJ, Hopman JCW, Klaessens J, van der Werf T, Daniëls O. The intra- and interoperator variability of Echo-Doppler measurements for noninvasive pulmonary arterial pressure determination in congenital heart disease. European Journal of Ultrasound. 1996; 4(2):91-98.

[24] Montorsi P, Cavoretto D, Alimento M, Muratori M, Pepi M. Prosthetic mitral valve thrombosis: can fluoroscopy predict the efficacy of thrombolytic treatment? Circulation 2003; 108(Suppl. II):79-84.

[25] Muratori M, Montorsi P, Teruzzi G, Celeste F, Doria E, Alamanni F, et al.Feasibility and diagnostic accuracy of quantitative assessment of mechanical prostheses leaflet motion by 
1 transthoracic and transesophageal echocardiography in suspected prosthetic valve dysfunction.

2 Am J Cardiol. 2006; 97:94-100.

3 [26] Celiento M, Filaferro L, Milano AD, Anastasio G, Ferrari G, Bortolotti U. Single center 4 experience with the Sorin Bicarbon prosthesis: a 17-year clinical follow-up. J Thorac Cardiovasc 5 Surg. 2014; 148(5):2039-2044.

6 [27] Azarnoush K, Laborde F, de Riberolles C. The Sorin Bicarbon over 15 years clinical 7 outcomes: multicentre experience in 1704 patients. Eur J Cardiothorac Surg. 2010; 38(6):7598766.

9 [28] Burstain JM, Brecher ME, Halling VW, Pineda AA. Blood volume determination as a function 10 of hematocrit and mass in three preservative solutions and saline. Am J Clin Pathol. 11 1994;102(6):812-815. 